\title{
Fermilab Test Beam Facility Annual Report: FY17
}

\author{
M. Rominsky ${ }^{1}$ \\ E. Schmidt ${ }^{1}$ \\ R. Rivera ${ }^{1}$ \\ L. Uplegger ${ }^{1}$ \\ J. Asaadi ${ }^{2}$ \\ J.L. Raaf ${ }^{1}$ \\ J. Freeman ${ }^{1}$ \\ J. Price ${ }^{3}$ \\ B. Casey $^{1}$ \\ R. Ehrlich ${ }^{4}$ \\ R. Belmont ${ }^{5}$ \\ S.Boose ${ }^{6}$ \\ M.Conners $^{7}$ \\ J. Haggerty ${ }^{6}$ \\ K. Hill ${ }^{5}$ \\ A. Hodges ${ }^{7}$ \\ J. Osborn ${ }^{9}$ \\ J. Huang 6 \\ E. Kistenev ${ }^{6}$ \\ J. Lajoie ${ }^{8}$ \\ E. Mannel ${ }^{6}$

\section{Skoby ${ }^{9}$} \\ C. Pontieri ${ }^{6}$ \\ M. Purschke ${ }^{6}$ \\ M. Sarsour ${ }^{7}$ \\ A. $\operatorname{Sen}^{8}$ \\ A. Ronzhin ${ }^{1}$
S. Stoll ${ }^{6}$ \\ F. Toldo ${ }^{6}$ \\ B. Ujvari ${ }^{10}$ \\ C. Woody ${ }^{6}$ \\ R. Demina ${ }^{13}$ \\ K. Hanagaki ${ }^{11}$ \\ A. Apresyan ${ }^{1}$ \\ T. Bose $\mathrm{B}^{12}$ \\ A. Canepa ${ }^{1}$ \\ E.Hazen ${ }^{12}$ \\ Y.Gershtein ${ }^{14}$ \\ E.Halkiadakis ${ }^{14}$ \\ M. Haytmyradov ${ }^{15}$ \\ M. Narain ${ }^{16}$ \\ O. Hindrichs ${ }^{13}$ \\ S. Korjenevski ${ }^{13}$ \\ J. Nachtman ${ }^{15}$ \\ B. Schneider ${ }^{1}$
K. $\mathrm{Nash}^{14}$ \\ Y.Onel ${ }^{15}$ \\ M. Osherson ${ }^{14}$ \\ D.Rankin ${ }^{12}$ \\ F. di Bello ${ }^{18}$ \\ B. Stone ${ }^{14}$ \\ J. Metcalfe ${ }^{17}$ \\ M. Benoit ${ }^{18}$ \\ M. Vicente ${ }^{18}$ \\ M. Kiehn ${ }^{18}$ \\ Rui Wang ${ }^{17}$ \\ L. Meng ${ }^{18}$ \\ E. Cavallaro ${ }^{19}$ \\ S. Chakanov ${ }^{17}$ \\ D. Frizzell ${ }^{20}$ \\ M. Zhang ${ }^{22}$ \\ T. Weston ${ }^{20}$ \\ A. Miucci $^{21}$ \\ L. Nodulman ${ }^{17}$ \\ S. Terzo ${ }^{19}$ \\ Junqie Xie $^{17} \quad$ L. $\mathrm{Xu}^{6}$ \\ E. Zaffaroni ${ }^{18}$ \\ M. Noulai ${ }^{23}$ \\ C. Argelles ${ }^{23}$ \\ S. Axani ${ }^{23}$ \\ J. Conrad ${ }^{23}$ \\ T. Katori ${ }^{24}$
L. Barner ${ }^{26}$
A. Grove 26
A. $\operatorname{Roth}^{26}$ \\ S. Mandalia ${ }^{24}$ \\ P. Sandstrom ${ }^{25}$ \\ J. Mohler ${ }^{26}$ \\ A. Kryemadhi ${ }^{26}$ \\ M. van Beuzekom ${ }^{27}$ \\ E. Dall'Occo ${ }^{27}$ \\ H. Schindler ${ }^{28}$ \\ W. Badgett ${ }^{1}$ \\ D. Denisov ${ }^{1}$ \\ S. Lukic ${ }^{29}$ \\ P. Ujićn ${ }^{29}$ \\ J. Paley ${ }^{1}$ \\ L. Fields ${ }^{1}$ \\ D. Christian ${ }^{1}$ \\ R. Zaki ${ }^{30}$ \\ P. L.G. Lebrun ${ }^{1}$ \\ ${ }^{1}$ Fermi National Accelerator Laboratory \\ ${ }^{2}$ University of Texas at Arlington \\ ${ }^{3}$ University of Liverpool \\ ${ }^{4}$ University of Virginia \\ ${ }^{5}$ Colorado University \\ ${ }^{6}$ Brookhaven National Laboratory \\ ${ }^{7}$ Georgia State University \\ ${ }^{8}$ Iowa State University \\ ${ }^{9}$ University of Michigan \\ ${ }^{10}$ Debrecen University \\ ${ }^{11} \mathrm{KEK}$ \\ ${ }^{12}$ Boston University \\ ${ }^{13}$ University of Rochester
}




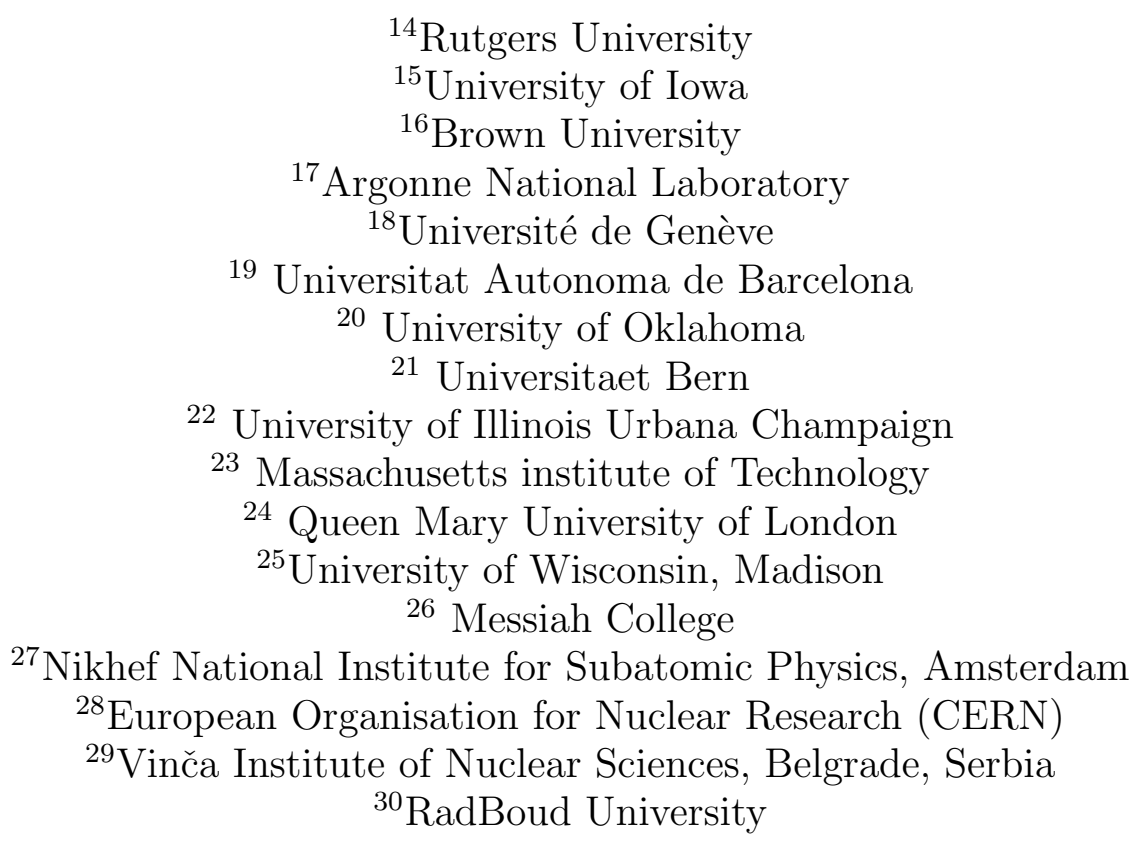

January 23, 2018

\begin{abstract}
This Technical Memorandum (TM) summarizes the Fermilab Test Beam operations for FY2017. It is one of a series of annual publications intended to gather information in one place. In this case, the information concerns the individual experiments that ran at FTBF and are listed in Table 1. Each experiment section was prepared by the relevant authors, and was edited for inclusion in this summary.
\end{abstract}

\title{
1 Summary of FY17
}

The Fermilab Test Beam facility continues to provide users with a place to characterize, calibrate, and develop new detector technologies. In FY17, we've worked to implement a number of improvements based on user feedback and recommendations from the committee. The following items were accomplished:

- The new Technical Scope or Work (TSW)/Operational Readiness Clearance (ORC) system has been in use for the last year. While it has improved signature approval time, the tool still has some functional bugs to work out.

- We have made progress on simulating both the MTest and the MCenter beam-line. 
- We installed a muon tagger for the MTest beam-line during the summer shutdown, which will be commissioned during the first weeks of beam.

- We made the virtual machine tools for offsite access more robust.

- We hosted 6 interns during the summer, as well as a non beam group of students testing a new type of tracker.

- Both MCenter and MTest were utilized during the full year.

During the FY17 year, our users were able to achieve their physics goals, prepare publications, and present data at conferences around the world. We consider this a very successful year. At one point, we had 5 experiments running at the same time in the MTest beam-line. This is a good example of our users working together to help each other achieve their research goals. We also supported several student groups over the summer. These groups did a variety of tasks aimed at teaching them skills with detectors and providing new tools for the Test beam.

\section{Research Overview at the Test Beam}

Each test beam experiment is required to prepare a Technical Scope of Work (TSW) with the Laboratory in which the beam, infrastructure, and safety requirements are spelled out in detail. Six new experiments were approved in FY17 and starting taking data. Ten experiments returned to continue their programs. These 16 experiments are listed in Table 1 and represent 108 collaborators from 30 institutions in a variety of countries. Figure 1 and Figure 2 show how the experiments broke down by research focus and user classification. Table 2 shows the raw numbers for professional groups, research focus (distinct Technical Scope of Work requests), and publications. FIgure 3 shows the type of beam requested on a weekly basis and is compared with the FY16 requests. We supported groups from both ATLAS and CMS. We used our high rate tracking area for a test by LHCb, who plan to continue to use the facility over the next few years. We hope to continue to use the High Rate Tracking area as well. We supported the sPHENIX collaboration from Brookhaven, and the Mu2e experiment tested a variety of detectors. We had several non beam users this year including g-2 which continued their rate of rise tests and a group of students from Mexico who were testing a new tracker. We fully supported the neutrino experiments LArIAT and LAToF for ProtoDUNE. We also had several groups come in to test general detector research and development. Full information is included in the second half of this report.

\section{$2.1 \quad$ Publications}

The groups at the test beam will often report their findings in internal notes, at conferences, and in published journals. This past year, all sixteen groups have plans for publications or 
have presented results at conferences. They list them in their sections below. In addition, several groups from the past have published results based on previous years' beam time. In total, there were 4 poster presentations, seven papers in preparation, and three papers published this year for last year's results. See Appendix A for the full list of publications in preparation, note that the individual reports also list these contributions.

- "Design and Beam Test Results for the sPHENIX Electromagnetic and Hadronic Calorimeter Prototypes", sPHENIX collaboration. Submitted to IEEE Transactions on Nuclear Science. arXiv:1704.01461

- "Photoelectron Yields of Scintillation Counters with Embedded Wavelength-Shifting Fibers Read Out with Silicon Photomultipliers". A. Artikov et. al (Mu2e CRV group). Submitted to NIM. arXiv:1709.06587

- "Modular focusing ring imaging Cherenkov detector for electron-ion collider experiments". X. He et. al. NIM-A

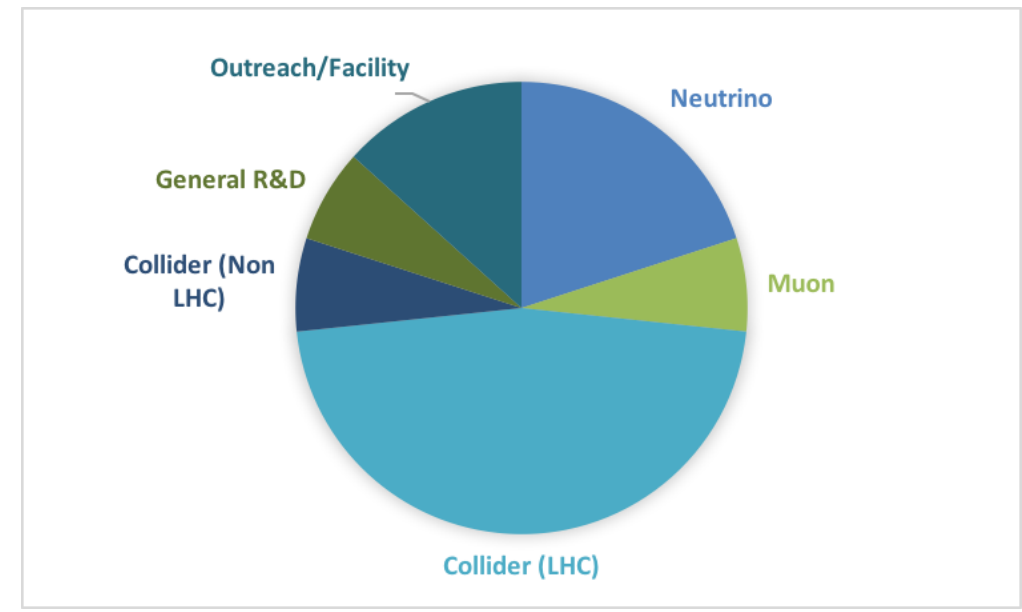

Figure 1: Breakdown of FY17 users by research focus. The test beam supports a broad range of areas. 


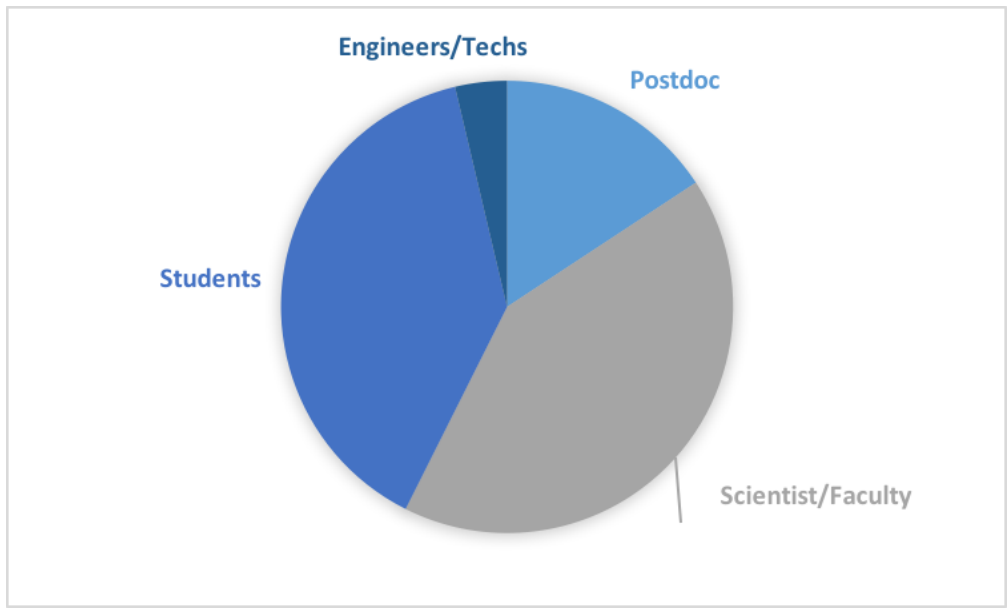

Figure 2: Breakdown of FY17 users by professional class.

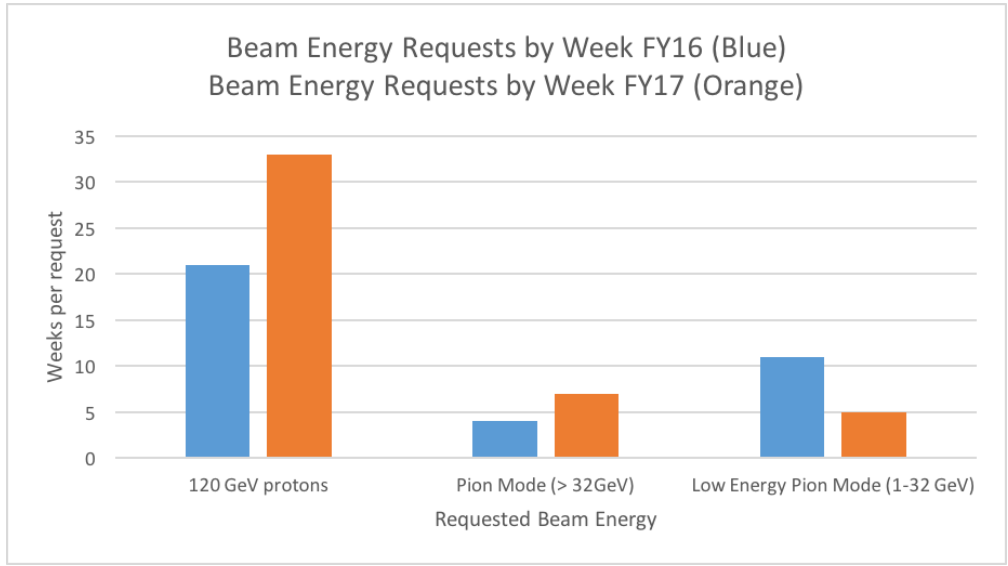

Figure 3: Requested beam energy by week for both FY17 and FY16. 


\begin{tabular}{|l|l|}
\hline Experiment Number & Description \\
\hline T0992 & Radiation Hard Sensors for the HL-LHC \\
T1034 & LArIAT: Liquid Argon in a Test Beam \\
T1041 & CMS Forward Calorimetry R\&D \\
T1042 & g-2 Straw Tracker Vacuum Tests \\
T1043 & Mu2e Cosmic Ray Veto Detector \\
T1044 & sPHENIX Calorimetry Tests \\
T1065 & Secondary Emitters Study \\
T1068 & Beam Tests of the SVX4 Telescope \\
T1209 & CMS Outer Tracker R\&D \\
T1224 & ATLAS Pixel Telescope Tests \\
T1267 & IceCube DOM Beam Test \\
T1272 & EM Mini Calorimeter \\
T1273 & LHCb VELO High Rate Test Beam \\
T1274 & LAFTBFToF For ProtoDUNE \\
T1314 & Muon Strips for Future Colliders \\
T1315 & LBNF Spectrometer: Prelim RF Bucket Occupancy Measurement \\
\hline
\end{tabular}

Table 1: Test Beam experiments performed in FY17.

\begin{tabular}{|l|l|}
\hline FTBF FY17 By the Numbers & \\
\hline Professional Class & Total \\
\hline Students & 44 \\
Professors/Scientists & 45 \\
Engineers/Technicians & 4 \\
Postdocs & 17 \\
\hline Experiment Focus (TSWs) & Total \\
\hline Muon & 2 \\
Neutrino & 3 \\
Collider (LHC) & 6 \\
Collider (Non-LHC) & 1 \\
General R\&D & 2 \\
Outreach/Facility & 2 \\
\hline Publications & total \\
\hline Journals & 2 \\
Conference proceedings/presentations & 3 \\
Posters & 4 \\
Conceptual Design/Technical Design reports & 2 \\
Internal Notes & 4 \\
\hline
\end{tabular}

Table 2: Statistics for Test Beam experiments performed in FY17. 


\title{
3 T992: Tests of Radiation-hard Sensors for the HL-LHC Motivation and Goals
}

\author{
R. Rivera, L. Uplegger
}

Beam used: $120 \mathrm{GeV}$ protons. Run dates: Nov 30-Dec 20, 2016, Feb 22- Mar 7, 2017, and Apr 26-May 9, 2017 This configuration (Figure 4) has been setup to study detector efficiency and time walk. At the HL-LHC, after $2500 \mathrm{pb}^{-1}$ of data, the expected maximum fluence for the pixel region $(<20 \mathrm{~cm})$ will be $2.5 \times 10^{16} \mathrm{neq} / \mathrm{cm}^{-2}$. To cope with this unprecedented radiation environment, there have been quite a few international collaborations formed to find possible solutions for vertex and tracking detectors at the HL-LHC. These include the RD42, RD49, and RD50 collaborations. A variety of solutions have been pursued. These include diamond sensors, 3D sensors, MCZ planar silicon detectors made from MCZ wafers, epitaxial, p-type silicon wafers and thin silicon detectors. The experimenters wish to compare the performance of this wide variety of detectors in a test beam before and after irradiation. To do so, the experimenters use the FTBF pixel and strip tracking telescopes which have $8 \mu \mathrm{m}$ resolution at the device under test. In particular, the experimenters are planning to study the charge collection efficiency of irradiated and un-irradiated devices and the spatial resolution as a function of the track incident angle. For the foreseen upgrade in luminosity CMS will also upgrade the strip tracker and the new Read Out Chip (ROC) will need to be tested in more realistic conditions than on a test bench.

\section{Setup}

The pixel and strip telescopes are read out through a custom DAQ system known as CAPTAN. A gigabit Ethernet board is used to route UDP data to a computer which is connected to a Fermilab server via internet. The readout boards are located close to the detector in the hut, and share a common clock and trigger signal. The detectors themselves may be operated up to $\approx 800 \mathrm{~V}$.

\section{Results and Publications}

The experimenters studied 3D silicon, new prototypes of planar sensors with a small pitch $(25 \mu \mathrm{m})$ and several n-on-p type silicon thin planar sensors. 3D tracking detectors and thin n-on-p sensors are promising radiation-hard candidates to replace planar n-on-n detectors in the HL-LHC. Radiation damage effects are measured with regards to charge collection, efficiency, and resolution of the particle tracks in beam tests, as well as leakage current and pixel noise. We have studied two varieties of $3 \mathrm{D}$ sensors: $1 \mathrm{E}, 2 \mathrm{E}$ (the number referring to 


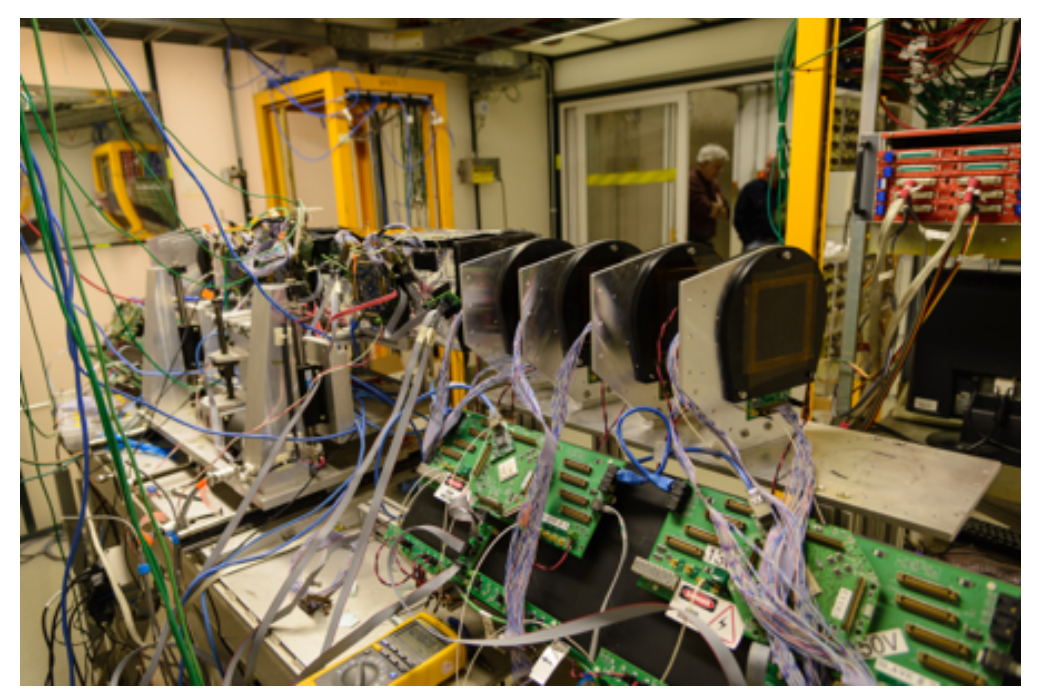

Figure 4: CMS Pixel telescope located in the MTest beamline, section 6.1a

the number of implant pattern per pixel), irradiated at CERN and in a neutron reactor in Ljubljana with doses up to $10^{16} \mathrm{neq} / \mathrm{cm}^{-2}$. Unfortunately, after irradiation, some devices that were irradiated with neutrons didn't work anymore but some others irradiated at CERN were still working collecting the expected amount of charge for the dose they have been irradiated at. The Fondazione Bruno Kessler (FBK), in Italy, fabricated these devices. Right now the accumulated dose is still limited by the readout chip but a new more radiation hard chip will be used for the upcoming test beam. We also tested several prototypes of thin sensors with n-on-p technology which are right now the preferred candidate by the CMS collaboration. These devices were also fabricated at FBK and they performed well giving high efficiencies even if they only generate one-third of the charge with respect to the traditional thicker sensors. After irradiation these devices still worked with a reduced, as expected by the effects of radiation, charge collection efficiency. In December 2016 and April 2017 the collaboration tested pixel-strip and strip-strip devices for the CMS phase II upgrade, using the same telescopes used for pixel tests. Measurements of time walk and detector efficiencies have been used for the Technical Design Report for those upgrades. New ROCs will be tested in upcoming test beams and will qualify the read out chain and module construction for the phase II upgrade. 


\title{
4 T1034: LArIAT
}

\section{Motivation and Goals}

\author{
J. Asaadi, J.L. Raaf
}

Beam used: 8-64 GeV $p i^{+/-}$

Run dates: November 2016 - July 2017

LArIAT (T-1034) is characterizing the performance of liquid argon time projection chambers (LArTPCs) in the range of energies relevant to short- and long-baseline experiments such as MicroBooNE, the SBN program, and DUNE. Measurements made in LArIAT aim to be useful for both neutrino physics and for proton decay searches. Measurements of charged pion and kaon interaction cross sections will be made, and these data will also be used to optimize particle identification capabilities. In addition, LArIAT will study energy resolution improvements that may be achievable by combining information from scintillation light and ionization charge signals. The data collected in LArIAT will also be used to study the possibility of determining the sign of charged particles (pions/muons) without a magnetic field.

\section{Setup}

The LArIAT setup is shown in Figure 5, located in the MCenter beamline at MC7. The incoming secondary beam was operated in both positive and negative polarity for a range of beam energies, and the tertiary magnets were also operated across their full range. The setup uses 4 multiwire proportional chambers (MWPCs) and 2 time-of-flight (TOF) scintillator paddles provided by FTBF. Additional beam instrumentation, such as aerogel Cherenkov counters, halo and punchthrough veto scintillator paddles, and muon range stack were put in place by the experiment. The heart of the experiment is a two-plane (240 wires each, with 4mm spacing in Run I and Run-II, and with 5-mm spacing in Run-IIIa and 3mm spacing in Run-IIIb) liquid argon time projection chamber (LArTPC) with 2 cryogenic PMTs and 3 SiPMs viewing the active volume. In Run-III, the experiment focused more on $R \& D$ for other experiments (SBND, DUNE) by installing prototype versions of two new detector design elements. For SBND, the LArIAT experiment installed a mesh cathode, which was operated with and without a wavelength-shifting reflector foil installed between two sheets of mesh. And for DUNE, LArIAT installed a prototype novel light collection device called ARAPUCA, which operates by trapping light inside a box, after it has passed through a dichroic filter. 


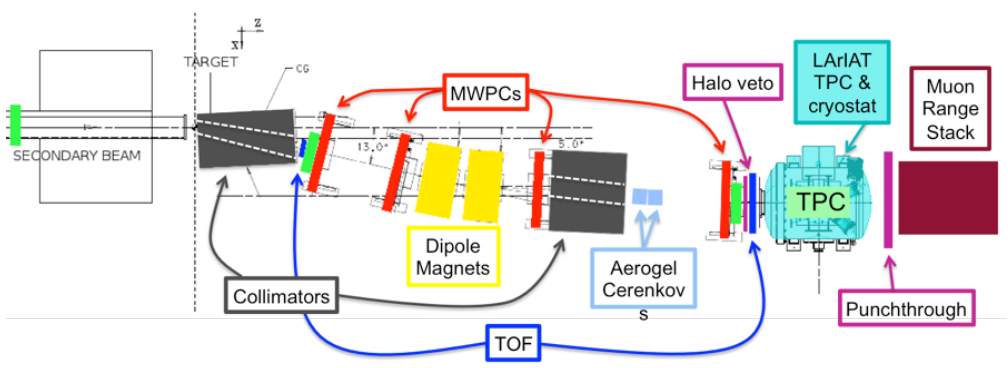

Figure 5: LArIAT layout in the MCenter beamline.

\section{Results and Publications}

One benefit of the mixed-particle beamline is the opportunity to collect particles that were not the main focus of the experiment. In this vein, we have a collection of approximately 100 anti-protons, which are currently the focus of study to ascertain the capabilities of LArTPCs to identify and reconstruct these types of interactions. This is relevant for DUNE, which will search for rare processes such as baryon-number-violating neutron-antineutron oscillation that is predicted by many grand unified theories. In n-nbar oscillation, the oscillated anti-neutron annihilates with a nucleon inside the nucleus, resulting in a final state with many pions. Anti-proton annihilation within the LArIAT detector gives a similar experimental signature (although the underlying physics is not identical). An example of one identified anti-proton candidate from the LArIAT Run-II data is shown in Figure 6.

In the past year, LArIAT has continued to analyze data from the Run-I and Run-II data-taking periods, focusing on finalization of the charged pion-argon total cross section measurement (updated to include Run-II data, which are much higher in both quality and quantity). In addition, the measurement of cross sections for various exclusive pion interaction channels are also underway, including pion charge exchange, pion absorption, and pion scattering. The kaon data are also under analysis, following the same ?thin-slice? technique as used in the pion analyses. We expect to have several analysis papers ready for publication this year.

Each of the cross section measurements will be useful for improving and validating Geant- 4 simulations, which can then be used for all future LArTPC experiments. These data will also be used to refine particle identification techniques in LArTPCs. The results will feed back to future LAr experiments, where a better understanding of the capabilities and efficiencies for identifying different particle species will lead to improved measurements of neutrino interactions. 


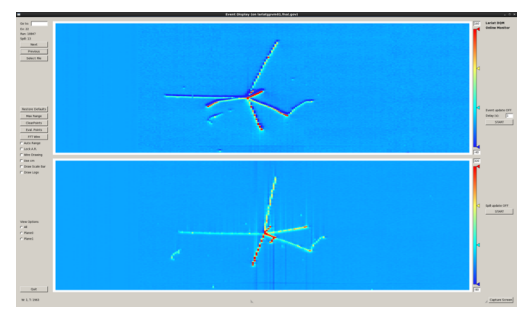

Figure 6: LArIAT Run II antiproton candidate event.

\section{T1041: CMS Forward Calorimetry R\&D}

\section{Motivation and Goals}

\section{J. Freeman}

Beam: $120 \mathrm{GeV}$ protons

Run dates: May 2017 to July 2017 (parasitic)

SiPM-on-tile is the baseline concept for the scintillator component of the CMS HGC upgrade calorimeter. Roughly one-half the sampling area will be scintillator and the other half will be silicon. The scintillator part will be segmented into small tiles, each read out by an SiPM. This test experiment studied light yield and uniformity of SiPM-on-Tiles for various choices of base material, size, edge treatment, thickness, wrapping material, and SiPM size. These studies will be used to better define the calorimeter design.

\section{Setup}

The test setup, shown in Figure 7, was a remote controlled $\mathrm{x}$-y stage with a small dark box attached. Inside the dark box was the sample SiPM-on-Tile. The SiPM was read out by a DRS4 waveform digitizer.

\section{Results and Publications}

The samples in Figure 8 were tested in this beam exposure:

For each sample uniformity and light yields were measured. An example a measurement at a single point on a sample is shown in Figure 9. The photo-electron peaks are clearly seen so the average light yield at each point is well determined.

Figure 10 shows data runs taken for for a $4 \times 4$ tile made of SCSN81, with a Tyvek wrapping. Each point represents a separate data run.

Figure 11 shows the light yield of the different points is shown below for $\mathrm{x}$ and $\mathrm{y}$ scans. The tile shows good uniformity. 


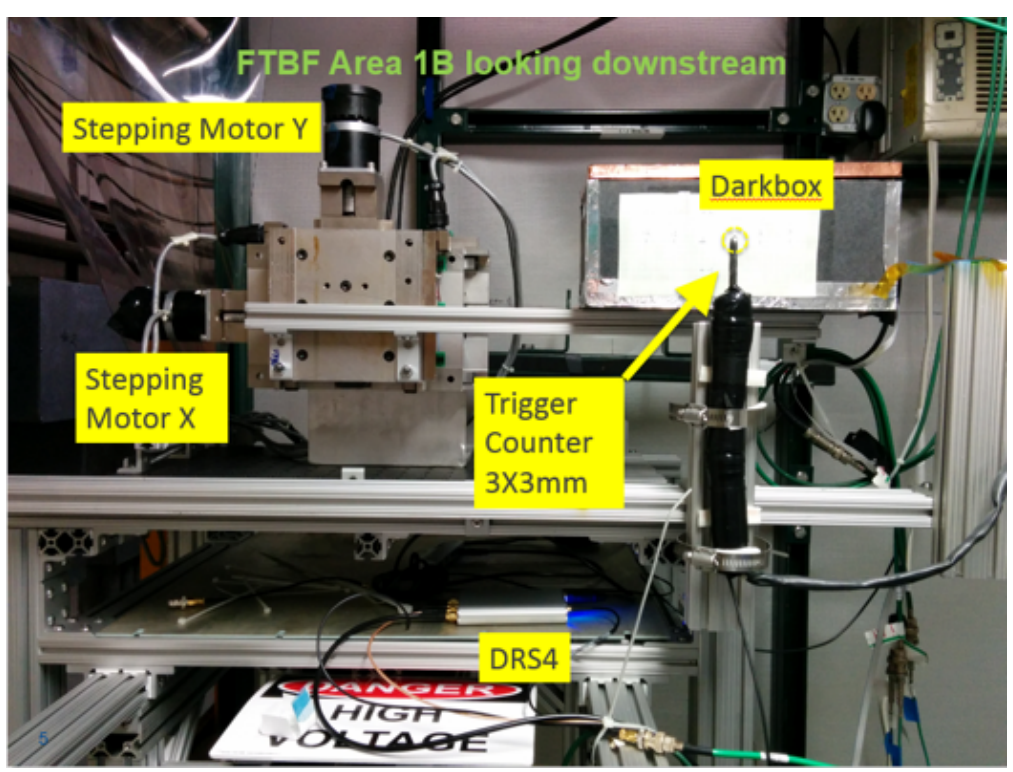

Figure 7: Setup for the T1041 experiment in MTest, section 6.1b.

$\begin{array}{lcccccc}\text { Sample } & \text { Transverse } & \text { Thickness } & \text { Cover } & \begin{array}{c}\text { Cover } \\ \text { big }\end{array} & \text { SiPM } \\ \text { side } & \mathrm{mm} \\ \text { S33Tyvek1.3 } & \text { Scintillator } & \mathrm{cm} & \mathrm{mm} & \text { edges } & & \\ \text { S44Tyvek1.3A } & \text { SCSN81 } & 3 \times 3 & 3.8 & \text { EJ-510 } & \text { Tyvek } & 1.3 \\ \text { LS33Tyvek1.3A } & \text { SCSN81 } & 4 \times 4 & 3.8 & \text { EJ-510 } & \text { Tyvek } & 1.3 \\ \text { S33WP1.3 } & \text { Siloxane } & 3 \times 3 & 3 & \text { EJ-510 } & \text { Tyvek } & 1.3 \\ \text { S33WP2.0 } & \text { SCSN81 } & 3 \times 3 & 3.8 & \text { EJ-510 } & \text { EJ-510 } & 1.3 \\ \text { EJ33ESR1.3 } & \text { SCSN81 } & 3 \times 3 & 3.8 & \text { EJ-510 } & \text { EJ-510 } & 2 \\ \text { S66Tyvek1.3 } & \text { EJ200 } & 3 \times 3 & 3.8 & \text { EJ-510 } & \text { ESR } & 1.3 \\ \text { SS66Tyvek2.0 } & \text { SCSN81 } & 6 \times 6 & 3.8 & \text { EJ-510 } & \text { Tyvek } & 1.3 \\ \text { EJ33Tyvek2.0 } & \text { SCSN81 } & 6 \times 6 & 3.8 & \text { EJ-510 } & \text { Tyvek } & 2 \\ \text { EJ33Tyvek1.3 } & \text { EJ200 } & 3 \times 3 & 3 & \text { EJ-510 } & \text { Tyvek } & 2 \\ \text { LS33Tyvek1.3B } & \text { EJ200 } & 3 \times 3 & 3 & \text { EJ-510 } & \text { Tyvek } & 1.3 \\ \text { NIU33Tyvek1.3 } & \text { Siloxane } & 3 \times 3 & 3 & \text { EJ-510 } & \text { Tyvek } & 1.3 \\ \text { S33Tyvek1.3_triple } & \text { Inject } & 3 \times 3 & 3 & \text { EJ-510 } & \text { Tyvek } & 1.3 \\ \text { S44Tyvek1.3_double } & \text { SCSN81 } & 3 \times 3 & 11.4 & \text { EJ-510 } & \text { Tyvek } & 1.3 \\ \text { EJ33MG01.3 } & \text { EJ200 } & 3 \times 3 & 3 & \text { MGO } & \text { MGO } & 1.3 \\ \text { S44Tyvek1.3B } & \text { SCSN81 } & 4 \times 4 & 3.8 & \text { EJ-510 } & \text { Tyvek } & 1.3 \\ \text { S33Tyvek1.3_double } & \text { SCSN81 } & 3 \times 3 & 7.6 & \text { EJ-510 } & \text { Tyvek } & 1.3\end{array}$

Figure 8: Samples tested in beam enclosure. 


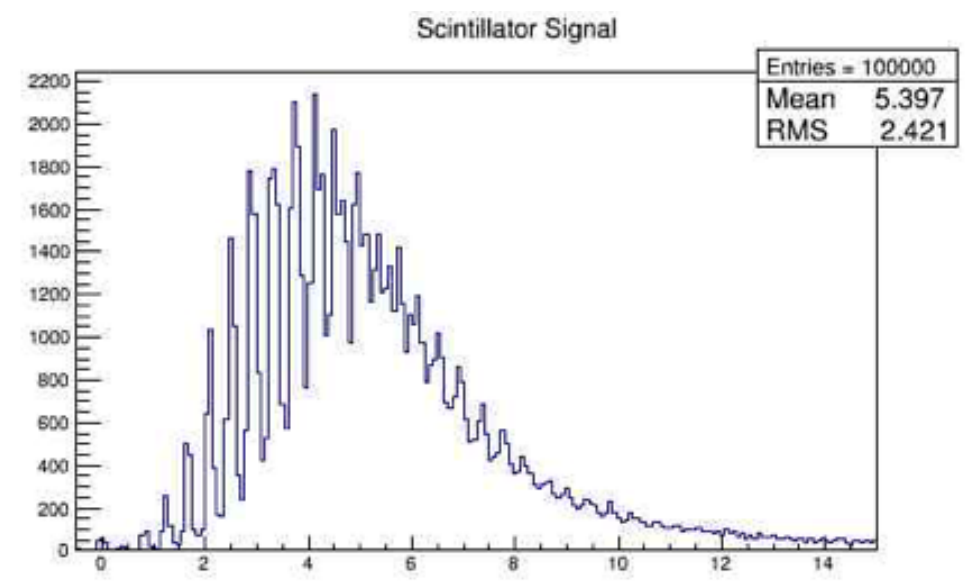

Figure 9: Example measurement for a single point.

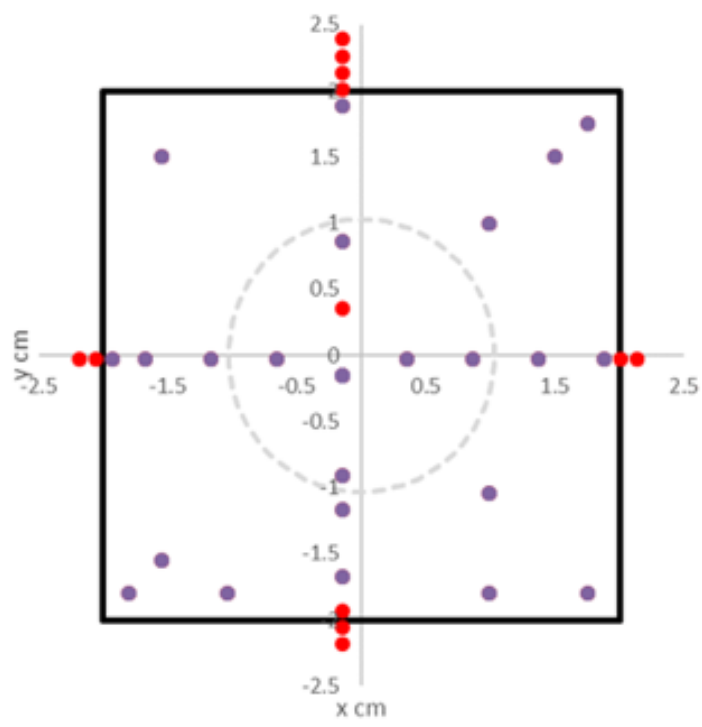

Figure 10: Results from a 4x4 tile made of SCSN81, with Tyvek wrapping. 

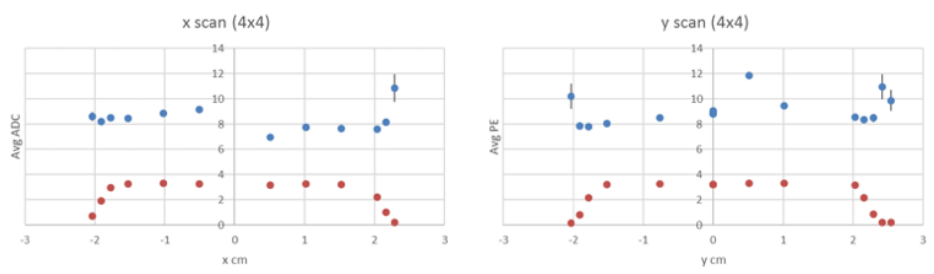

Figure 11: Light yield of different points in $\mathrm{x}$ and $\mathrm{y}$ scans.

The following chart( 12 shows light yield for different choices of wrapper material.

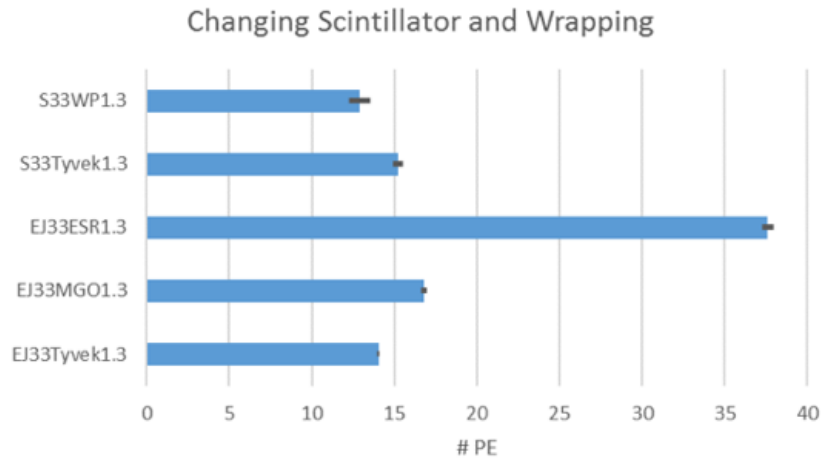

Figure 12: Shown are white paint covering, MGO covering, Tyvek, and ESR film. It is clear that ESR has a much higher light yield.

These and other results have been collated and are documented in CMS note: https: //cms-docdb.cern.ch/cgi-bin/DocDB/ShowDocument?docid=13393 


\section{T1042: G-2 Straw Tracker Test}

\section{Motivation and Goals}

\section{B. Casey, J. Price}

Beam used: No beam

Run dates: September 1st 2016 to August 31st 2017

The aim of this test is to measure the leak rate of a single module from the straw trackers to be used in the g-2 experiment, and ensure that the modules did not develop any leaks when being transported from Liverpool in the UK. The leak rate for a single module must be kept low enough for the required vacuum. Before the module assembly the leak rate of the straws is measured using $\mathrm{CO} 2$, and in the experiment Ar-C2H6 will be used. For safety reasons Nitrogen is mainly used a M-Test, however the Ar-C2H6 line is available for more accurate testing. The option to cool the gas is also available, as the permeation rate changes with temperature.

\section{Setup}

The module was placed inside the vacuum tank, and connected up to the gas supply, as shown in Figure 13. The turbo vacuum pump is connected to the vacuum tank. Background measurements without the module present are also made, and the temperatures and pressure in the room monitored since they can affect the rate of rise. A valve was placed on the gas input and output to isolate the system when pulling vacuum on the straws. To perform the rate of rise tests the vacuum pump valve is closed and the pressure inside the vacuum tank is read out by the connected gauge at a rate of $1 \mathrm{~Hz}$ for 600 seconds. This test is repeated for all modules as they arrive.

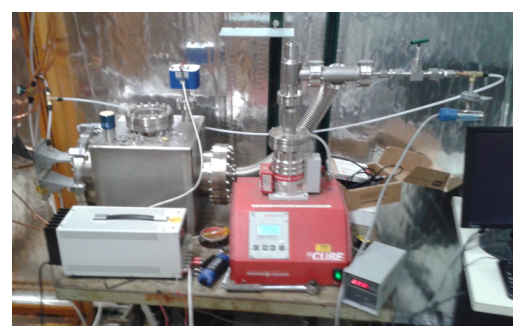

Figure 13: Setup at MTest in the gas room. the module is placed inside the vacuum tank and pumped down using the Pfeiffer turbo vacuum pump. The gas is supplied from MTest. 


\section{Results and Publications}

The results are shown in Figure 14. The fact that the vacuum level changes with temperature has allowed us to determine that the out gassing is a large component of the total vacuum level, and we can now correct the rate of rise values that we obtain for each tracker module for the temperature in the room that day. A full set of 8 tracker modules have been installed into the g-2 ring, and no leaks have been observed. A further 2 modules have already been tested, and 6 more will be done before November this year in time for the full data taking of the g-2 experiment.

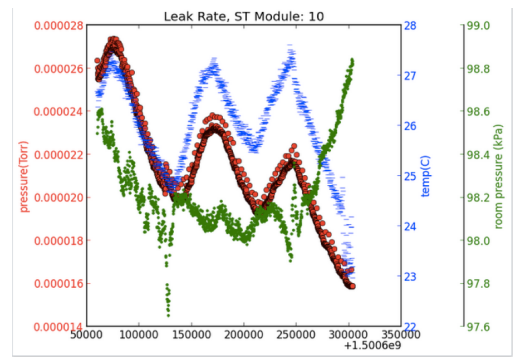

Figure 14: The pressure as measured by the vacuum gauge is plotted against time and is shown together with the temperature and pressure. There is a clear correlation with the pressure in the room and the vacuum level. The vacuum valve was kept open for this entire time. 


\section{T1043: Mu2e CRV Test Beam}

\section{Motivation and Goals}

R. Ehrlich

Beam used: $120 \mathrm{GeV}$ protons

Run dates: June 2017

Several configurations of prototype scintillation counters for the Mu2e Cosmic Ray Veto (CRV) detector were tested. The $3 \mathrm{~m}$ long counters were made of extruded plastic scintillator and contained 2 WLS fibers (1.4 mm diameter) readout by $2 \mathrm{~mm}$ x $2 \mathrm{~mm}$ SiPMs on both ends. Two counters are glued together to form a discounter. The goals of the test beam are summarized in the list below.

- Measure the photoelectron (PE) yield at various locations at the CRV dicounters (longitudinal, transverse, and angular scans)

- to verify that the required PE yield can be achieved, and

- to tune the CRV counter simulation.

- Measure PE yields close to the readout ends.

- Test radiation damaged SiPMs.

- Test silicone filled fiber channels.

- Test SiPMs coupled with optical grease.

- Test counters with/without reflectors.

- Test counters with/without white paint.

- Test the response for different SiPM biases.

\section{Setup}

Dicounters were tested four at a time as shown in Figure 15. Front End Board (FEB) readout electronics were mounted above the dicounters and data was sent to the control room through ethernet connections. XY scans were done by remote operation of the movable table and the mounting frame could be manually tilted for angular scans. 


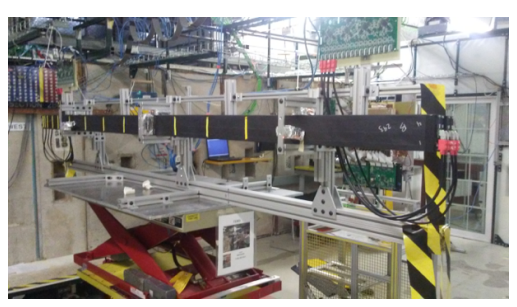

Figure 15: Photo of T-1043 setup showing CRV dicounters mounted on the moveable table.

\section{Results and Publications}

Figure 16 shows the PE yield distribution for a proton beam which was directed at a CRV counter $1 \mathrm{~m}$ away from the readout. The most probable PE value of both SiPMs (of one side of a counter) combined is 142.5 PEs. This exceeds the required PE yield. Publication of the full test beam results is pending.

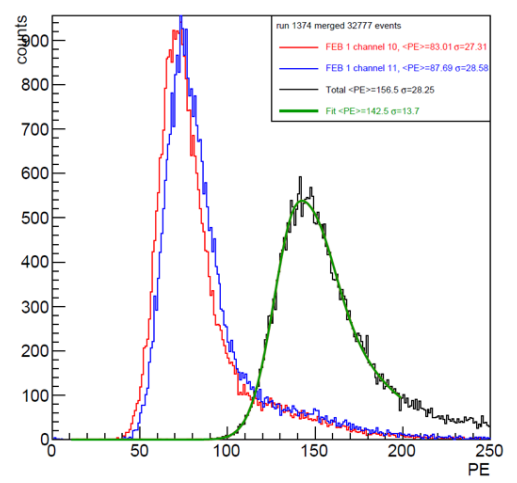

Figure 16: The pressure as measured by the vacuum gauge is plotted against time and is shown together with the temperature and pressure. There is a clear correlation with the pressure in the room and the vacuum level. The vacuum valve was kept open for this entire time. 


\section{T1044: sPHENIX Calorimeter Test}

\section{Motivation and Goals}

R. Belmont, S.Boose, M.Conners, J. Haggerty, K. Hill, A. Hodges, J. Huang, E. Kistenev, J. Lajoie, E. Mannel, J. Osborn, C. Pontieri, M. Purschke, M. Sarsour, A. Sen, M. Skoby, S. Stoll, F. Toldo, B. Ujvari, C. Woody

Beam used: Mixed Mode Pions/Primary Proton

Run dates: Jan 16, 2017 to Feb 23, 2017

The goal of the 2017 T-1044 running was to test the high rapidity sections of the sPHENIX electromagnetic and hadronic calorimeters along with upgraded frontend electronics and compare the energy response to Monte Carlo simulations.

The EMCal is a 2-D projective SpaCal design with scintillating fibers embedded in a tungsten powder infused epoxy. The HCal is a tilted steel plate with scintillating tiles design. Read out of both calorimeters uses a common set of electronics based on SiPMs as the optical sensors and having the amplified and shaped analog waveforms digitized with a $60 \mathrm{MHz}$ sampling frequency.

\section{Setup}

The detectors were located on a table in the MT6.2D section of the FTBF beam line as shown in Fig 17. The detectors were oriented to simulate charged particles from the striking the front of the high rapidity sections of the sPHENIX calorimeter system. The detectors were readout using the prototype frontend sPHENIX calorimeter electronics and waveform digitized at a $60 \mathrm{MHz}$ sampling frequency. The resulting data along with trigger information, and data from Cherenkov system was recorded using the PHENIX Data Acquisition system. Data was collected using pion beams from 1 to $60 \mathrm{GeV}$, both polarities and $120 \mathrm{GeV}$ primary protons.

\section{Results and Publications}

Analysis of the data collected during the 2017 running is ongoing to determine the energy resolution of the EMCal and $\mathrm{HCal}$ Calorimeters as a combined system. Figure 18 shows the energy response of the EMCal for $2 \mathrm{GeV} / \mathrm{c}$ electrons. The electrons were selected using the 2 beam line Cerenkov counters. The results are being prepared for publication in the Fall of 2017. 


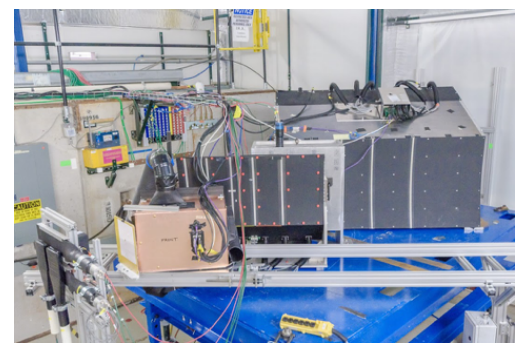

Figure 17: The sPHENIX high rapidity calorimeter modules located in the FTBF beam line. The EMCal module is in the front left, with the inner and outer HCal modules behind it.

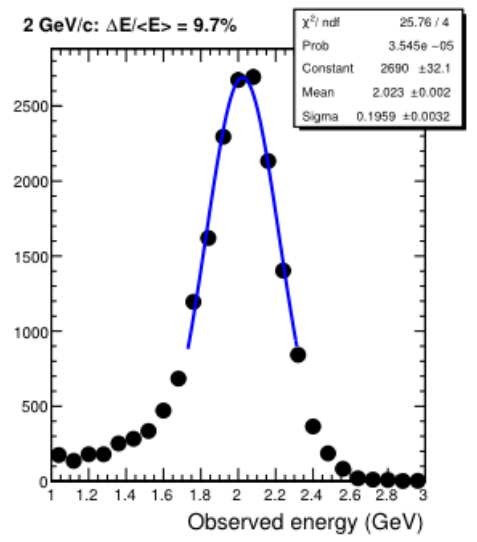

Figure 18: The EMCal energy response for $2 \mathrm{GeV} / c$ electrons. 


\section{T1065: Fast Timing Detectors}

\section{Motivation and Goals}

\section{A. Ronzhin}

Beam used: Low energy mixed particle mode

Run dates: May 10 to May 26, 2017

We prepared and built a Time of Flight (ToF) system with good timing resolution and a small material budget to provide the facility with. This will include a fast, thin, start counter to be used as trigger for other experiments.

\section{Setup}

The experiment was setup between the $6.2 \mathrm{~b}$ and $6.2 \mathrm{c}$ areas in MTest.

\section{Results and Publications}

The results are shown in Figure 19. The experimenters are preparing a NIM article.
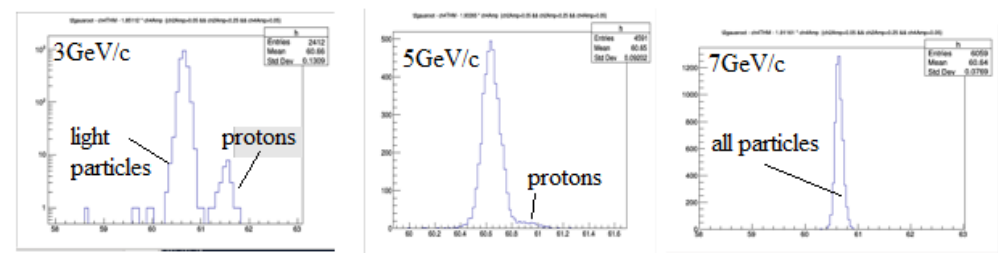

Figure 19: At $3 \mathrm{GeV}$ momentum there is clearly separation of protons from lighter particles. The is a 50 ps timing resolutions on the base distance of 5.6 meters 


\section{T1068: Beam Test of the SVX4 Telescope Motivation and Goals}

\section{K. Hanagaki}

Beam used: protons $120 \mathrm{GeV}$

Run dates: $1 / 18 / 2017-2 / 7 / 2017$

The purpose of this experiment is to study the silicon strip detector consisting of the silicon strip sensors and SVX4 readout ASICs, called SVX4 telescope, and to use this device as the reference detector to measure the charged particle incident position to the other detector under test (DUT). In addition, we tested the newly developed monolithic pixel sensors based on Silion-On-Insulator (SOI) technology. The goal is to confirm that the SOI sensor achieves the position resolution of order of 3 micron or 1 micron depending on the pixel sizes.

\section{Setup}

We have two independent setups, one with the SVX4 telescope and the prototype pixel sensors for the ATLAS Upgrade as the DUT, another consisting of six SOI sensors (Figure 20). There are two types of SOI sensors, FPIX whose pixel size is 8 micron squares, and SOFIST whose pixel size is 20 micron squares. The resolution studies for the SOI sensors are conducted by the cross reference method. Both setups use the FPGA based DAQ system.

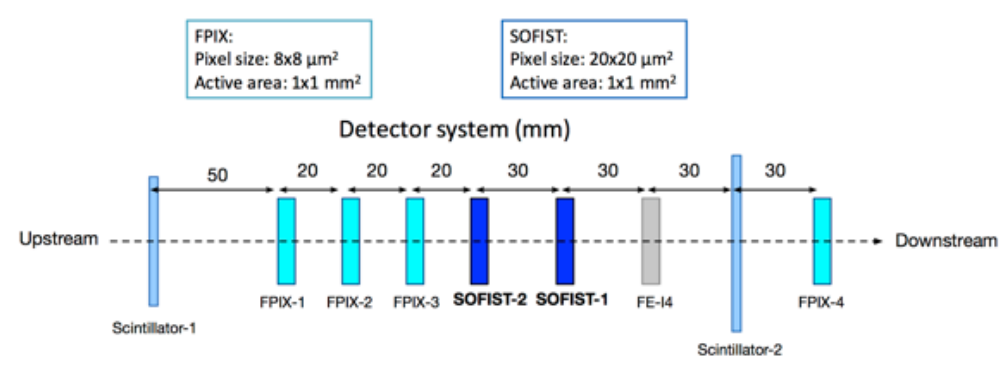

Figure 20: Setup for the SOI sensor testing. 


\section{Results and Publications}

By the cross calibration, the FPIX achieved position resolution better than 1 micron. By making use of this superb resolution, we used the FPIX as the telescope for the SOFIST. Figure 21 shows the residual distribution of one of the SOFIST sensors. It achieved the position resolution better than 1.3 micron although the pixel size is 20 micron. This is thanks to the capability of recording the analog information by ADC with the charge sharing for more than one pixel. In addition this measurement can be possible only with high momentum beam such as $120 \mathrm{GeV}$ proton at FTBF to reduce the multiple scattering.

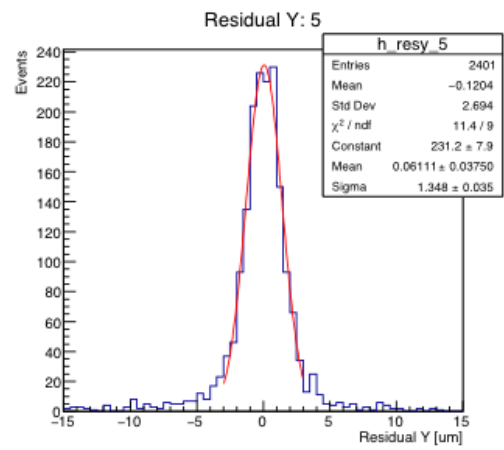

Figure 21: Residual distribution of SOFIST. 


\section{T1209 CMS OuterTracker}

\section{Motivation and Goals}

A. Apresyan, T. Bose, A. Canepa, R. Demina, Y.Gershtein, E.Halkiadakis, M. Haymyradov, E.Hazen, O. Hindrichs, S. Korjenevski, J. Nachtman, M. Narain, K. Nash, Y.Onel, M. Osherson, D.Rankin, B. Schneider, B. Stone

Beam used: Protons $120 \mathrm{GeV}$

Run dates: Jan 11-17, 2017

The goal of the experiment is to test the prototypes of the CMS Outer Tracker detectors, verifying performance of the individual components as they become available.

\section{Setup}

The experiment used a GLIB-based DAQ, integrated with the pixel telescope, show in Figure 22.

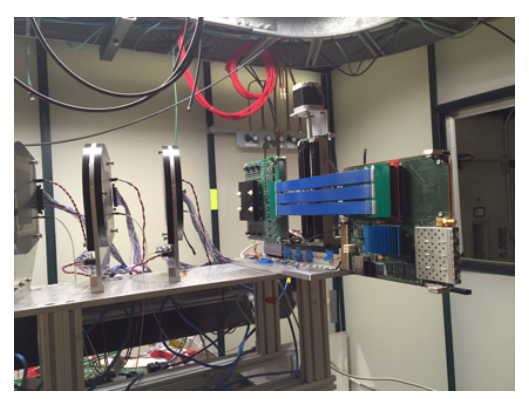

Figure 22: CMS Pixel telescope (facility instrument) and the CMS Outer Tracker detector (blue cables)

\section{Results and Publications}

The tests for the prototype of the Outer Tracker ROC include:

- Measured timewalk

- Demonstrated chip-to-chip communication and hit pair (stub) formation on the front end (necessary feature for track finding at $40 \mathrm{MHz}$ ) 


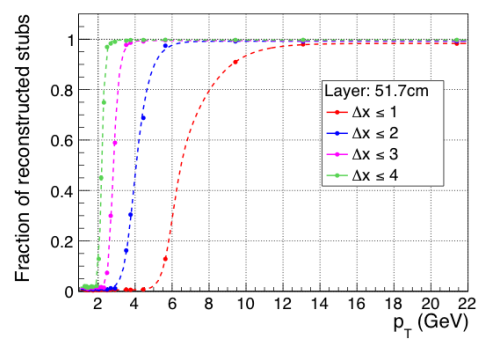

Figure 23: Stub efficiency v.s. emulated track momentum

\section{T1224: ATLAS Pixel}

\section{Motivation and Goals}

J. Metcalfe, M. Benoit, M. B. P. Vicente, F. di Bello, E. Cavallaro, S. Chakanov, D. Frizzell, M. Kiehn, L. Meng, A. Miucci, L. Nodulman, S. Terzo, R. Wang, T. Weston, J. Xie, L. Xu, E. Zaffaroni, M, Zhang

Beam Used: $120 \mathrm{GeV}$ protons, $60 \mathrm{GeV}$ mixed particle beam

Run Dates: February 22, 2017 to April 25, 2017

The main devices under test (DUTs) for this experiment are high voltage CMOS (HVCMOS) sensors, a candidate technology for the ATLAS Phase-II ITK Pixel Upgrade. A standard CMOS process is modified to provide a deep n-well with high resistivity that can be biased to form an active region sensitive to charged particles.

The sensors are H35demo prototypes and have 4 different pixel matrix types to compare performance. An amplification circuit is directly incorporated into two of the matrices and amplifier plus digitizer in the other two matrices. They were also produced in several different silicon resistivities. Additionally, a subset of samples were measured in the test beam, then sent for gamma irradiation, and then re-measured in the test beam to evaluate the radiation damage effects.

\section{Setup}

The HVCMOS test beam was conducted between February 22nd and April 25th. Typically we used a $120 \mathrm{GeV}$ proton beam with the maximum number of particles reaching around 3 million per spill. A pixel telescope was used to reconstruct tracks and then compare to the hits on the DUT. There were 6 planes of pixels with varying orientation to maximize the spatial resolution of the telescope. A cold box was available for the DUT that used air cooled by a chiller to reduce the temperature of the DUT. Details of this setup are 

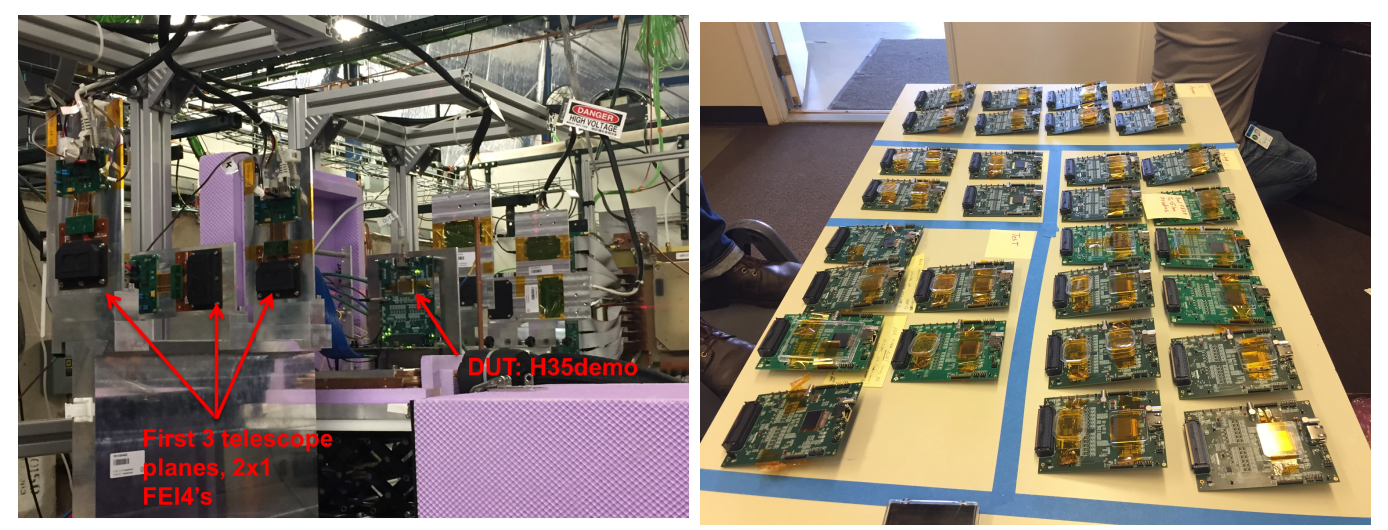

Figure 24: Left: The test beam setup used for the silicon CMOS studies includes 6 planes of pixel detectors and a cold box for the DUT. Right: An array of some of the H35demo samples laid out after testing.

shown in Figure 24. The telescope was placed on the motion table in section MT6.2b. The setup utilized nitrogen bottles provided by the FTBF to circulate nitrogen in the cold box to prevent condensation. The services of support personnel were utilized for making mechanical parts to adjust the position of the telescope and to install a large current converter needed to run the chiller.

\section{Results and Publications}

Over 60 samples were tested in the test beam. Many of the CMOS samples had an issue with a gradient in the glue thickness along the sensor causing non-uniform efficiencies across the sensor. This modified the capacitive coupling of each pixel limiting the direct comparison of results and reducing the expected wide array of performance studies. Figure 25 illustrates an extreme example of the gradient. A high efficiency is found in the upper right corner and then drops to $0 \%$ efficiency. This problem was addressed after the test beam for subsequent studies that are ongoing. Preliminary results from the test beam on the best samples indicate an efficiency above $99 \%$ is achievable demonstrating the potential of the technology. Two of the matrices in the CMOS sensor were fully digitized and did not rely on the capacitive coupling. The preliminary results from these studies show approximately 98\% efficiency shown in Figure 25.

A MIMOSA pixel sensor with $18 \mu \mathrm{m}$ pixels was integrated successfully into the telescope DAQ framework. This will provide a much higher spatial resolution in future runs with this telescope. Several planar silicon modules for the ATLAS Phase II pixel upgrade were evaluated for the bump-bond reliability as part of the qualification procedure before construction. A few samples were also tested in the test beam and then sent to Argonne 

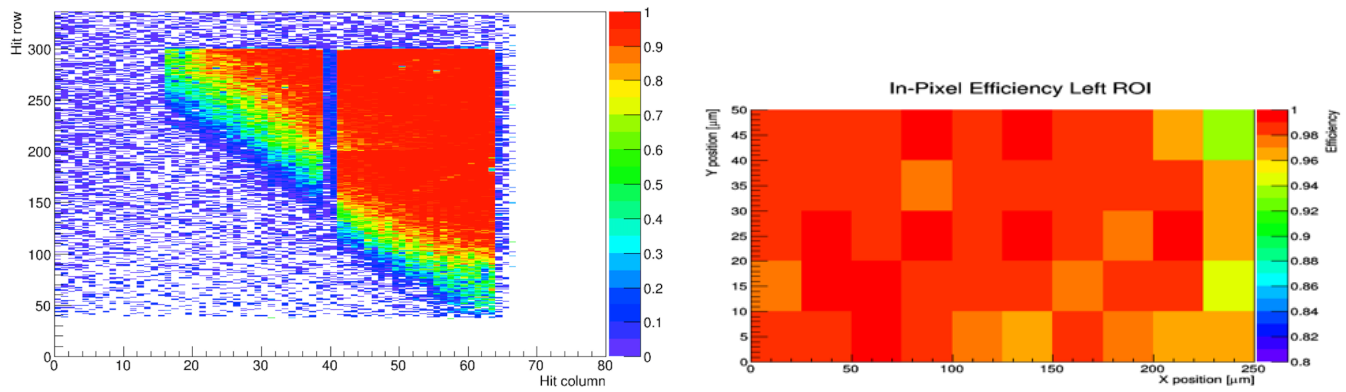

Figure 25: Left: An example of the tracking efficiency map with a gradient in efficiency due to a gradient in the glue thickness. Right: The efficiency of the fully digital CMOS matrix with an average of $98 \%$ tracking efficiency.

for gamma irradiation with doses up to $100 \mathrm{Mrad}$ and then re-tested in the test beam. A full list of resulting publications and presentations is listed below.

\section{Publications and presentations:}

- RTI Pixel Module Evaluation, ATLAS internal note, in preparation.

- Testbeam measurements of pixel sensor technologies for ITK upgrade, Dylan Frizzell, presentation at the US ATLAS Workshop, Argonne National Laboratory, July 2017.

- Track Reconstruction Efficiencies with the H35DEMO HV-CMOS Pixel Detector, Matt Zhang, presentation at the American Physical Society Division of Particles and Fields, Fermi National Accelerator Laboratory, August 2017.

- Track Reconstruction Efficiencies with the H35DEMO HV-CMOS Pixel Detector, Dylan Frizzell, poster at the American Physical Society Division of Particles and Fields, Fermi National Accelerator Laboratory, August 2017. 


\title{
16 T1267: IceCube DOM Test
}

\section{Motivation and Goals}

\author{
C. Argelles, S. Axani, J. Conrad, T. Katori, M. Noulai, S. Mandalia, P. Sandstrom
}

Beam used: $-2,4,8 \mathrm{GeV}$ negative pion mode, $2 \mathrm{GeV}$ muon mode, $120 \mathrm{GeV}$ proton mode Run dates: June $14-27,2017$ The motivation is to prove a new idea of particle identification (PID) using a single large photo-cathode PMT by analyzing pulse shape. A simulation shows it is possible to distinguish electromagnetic-like Cherenkov radiation (electron, photon) and MIP-like Cherenkov radiation (muon, pion) from the pulse shape of a single hit around few $\mathrm{GeV}$ region. This may be useful for current and future large water or ice Cherenkov detectors, such as IceCube and Hyper-Kamiokande.

\section{Setup}

We installed a large polyethylene food-quality tank (1010 gallon), filled with distilled water, coated with black tedlar both inside and outside. One IceCube digital optical module (DOM), consisting of a 10 inch PMT is enclosed in a glass shell, is supported by a floating mount on the water surface. The DOM photo-cathode is looking downward in order to observe Cherenkov radiation from the beam penetrating the tank. Pulse of each event is recorded by fast analog to digital convertor (ADC). The DOM is calibrated by a LED to monitor the gain, also beam spread is monitored by MWPC. Finally, four scintillation counters are used to trigger the signal.

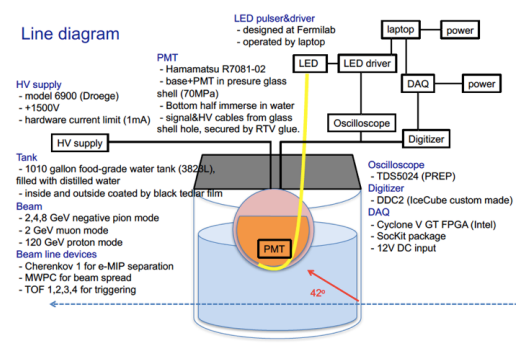

Figure 26: Setup of the experiment.

\section{Results and Publications}

We have not analyzed pulse shape distribution yet, but the collected charge distribution clearly shows different widths for MIP and electron peaks (Figure 27). This indicates 
potential PID of high energy MIP and low energy electron, where both provide same charge and traditional PID cannot distinguish. A positive result of this will be published in NIM or JINST, and can be used in IceCube simulation for an additional information of PID.

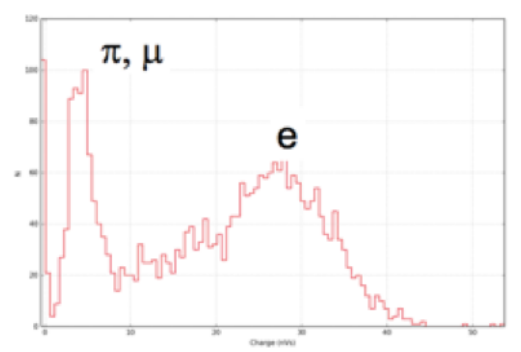

Figure 27: Results of the test beam experiment. 


\title{
17 T1272: Prototype EM Calorimeter
}

\section{Motivation and Goals}

\author{
A. Kryemadhi, L. Barner, A. Grove, J. Mohler, A. Roth
}

Beam used: $e^{+}$and $\pi^{+}(1-16 \mathrm{GeV})$

Run dates: March 13-17, 2017

The experimenters tested the performance of a compact mini calorimeter using LYSO crystals and Silicon-Photomultipliers with the goals of understanding dynamic range and construction of highly granular calorimeters for $\mathrm{GeV}$ gamma rays in space.

\section{Setup}

Figure 28 shows the readout board and the crystals on top of the board. The trigger consisted of three scintillator counters and a Cerenkov counter with pressure tuned for positrons.

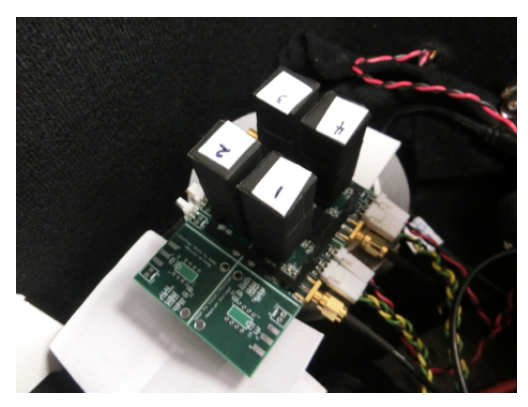

Figure 28: Readout Board with LYSO crystals on top of SiPMs.

\section{Results and Publications}

A compact detector with good granularity $(\approx$ one radiation length per tower $)$ was constructed and tested at Fermilab Test Beam Facility using 2-16 GeV range positrons. The small volume of the mini-calorimeter allowed for the study of the readout circuit, construction of mini-towers, dynamic range, and light yield. Detector response is linear up to $8 \mathrm{GeV}$ as shown in Figure 29. Light Yield for our mini calorimeter in the energy range 2-8 GeV is 1500 to 2300 photoelectrons when we used a Neutral Density Filter with OD

$=1$ (10\% Transmission), this number is much smaller than number of microcells in the SiPM arrays, indicating that these SiPM arrays would be well suitable to construct a full 
scale EM calorimeter for Astroparticle physics in space in GeV range. With a much better readout scheme we can extend the dynamic range.

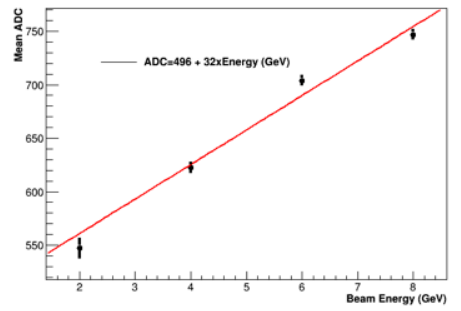

Figure 29: Detector response for different beam energies. 


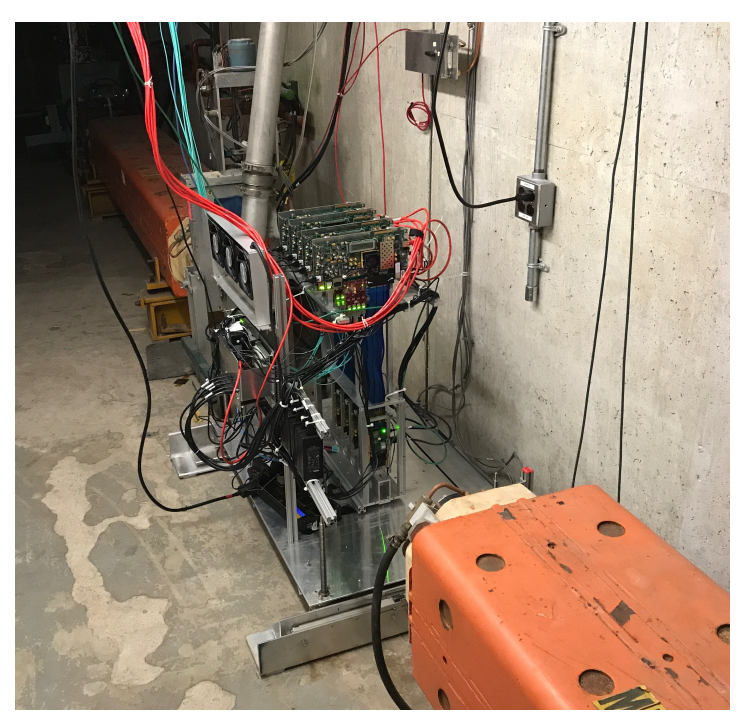

Figure 30: Photograph of the telescope as installed in the M03 high-rate area.

\title{
18 T1273: LHCb VELO Beam Test
}

\section{Motivation and Goals}

\author{
M. van Beuzekom, E. Dall'Occo, H. Schindler
}

Beam used: $120 \mathrm{GeV}$ Protons

Run Dates: May 3, 2017 to May 9, 2017

The LHCb upgrade, planned for 2019/20, will transform the data acquisition and trigger architecture of the experiment to $40 \mathrm{MHz}$ readout and fully software-based event selection. For the upgraded LHCb Vertex Locator (VELO), a pixel ASIC (dubbed "VeloPix") featuring data-driven readout at a rate of up to $800 \mathrm{MHits} / \mathrm{s}$ has been developed. The first version of the ASIC became available in autumn 2016, and the objective of this beam test was to evaluate its rate capability.

In order to measure the hit finding efficiency as function of the track (or particle) rate, a small beam telescope comprised of five planes of hybrid pixel detectors $(200 \mu \mathrm{m}$ thick $n$-on- $p$ silicon sensors with an active area of $\sim 1.4 \times 1.4 \mathrm{~cm}^{2}$, bump-bonded to VeloPix ASICs) was constructed. 

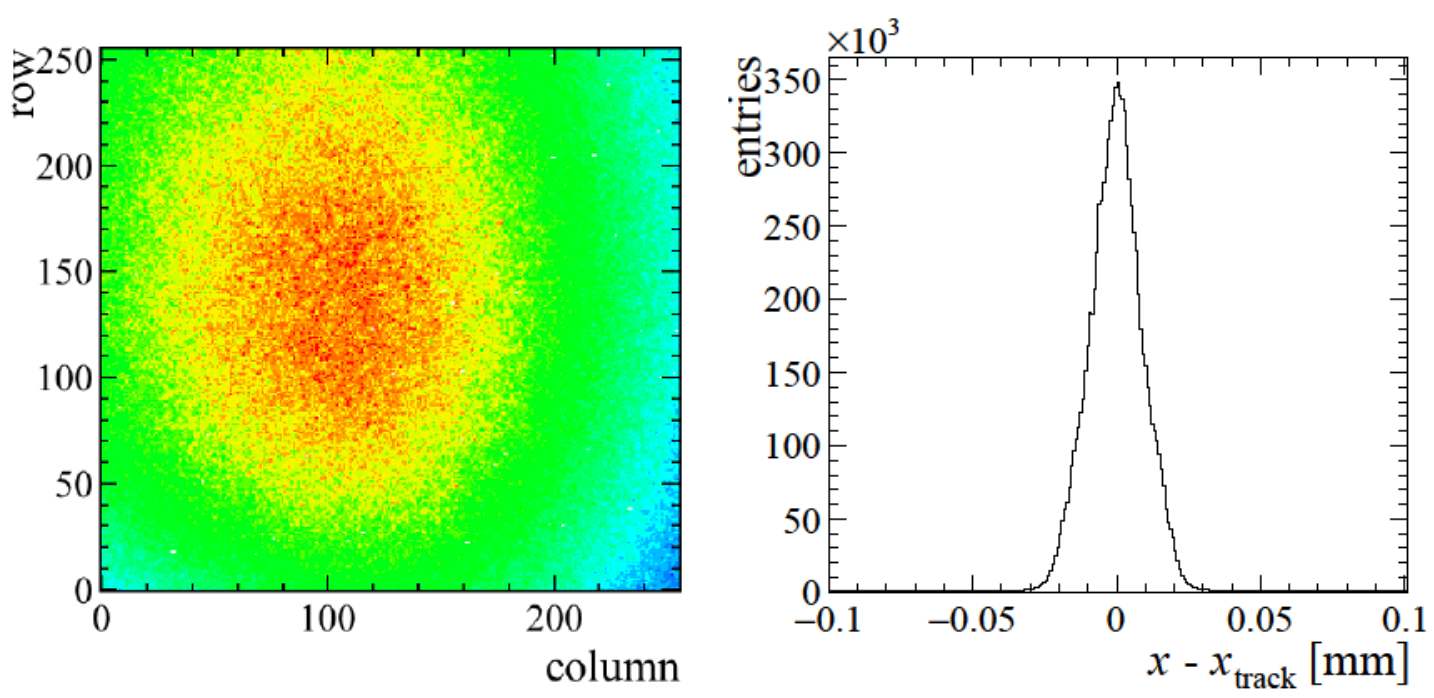

Figure 31: Hit map (left) and residual distribution in the horizontal direction (right).

\section{Setup}

The setup was installed in the M03 high-rate area on May 4th, followed by four days of data-taking. The beam line was operated in "Low Energy Pion" mode, i. e. using a $120 \mathrm{GeV}$ proton beam in M03.

A photograph of the setup as installed in the beam line is shown in Fig. 30. The mechanical frame of the telescope, holding the chipboards and the readout electronics, was mounted on the remote-controlled moveable table available in the zone. The readout electronics, implemented using commercial off-the-shelf FPGA development kits, were placed at a distance of $\sim 0.5 \mathrm{~m}$ above the active area of the telescope. From these readout boards, the data were sent over optical fibres to data acquisition PCs located at a distance of $\sim 10 \mathrm{~m}$ from the setup.

\section{Results and Publications}

Figure 31 shows the beam profile as seen by one of the telescope planes (the pixel pitch is $55 \mu \mathrm{m}$ in both the column and row direction). The basic telescope performance metrics are in agreement with expectations. As an example, Fig. 31 (right) shows the residual distribution in the horizontal direction for one of the telescope planes. Data were taken at intensities up to several 100 million hits per second. The detailed analysis of the data is ongoing, and a report is in preparation. 


\title{
19 T1274: Large-Area Fast Timing Beam Time of Flight (LAFTBToF)
}

\section{Motivation and Goals}

\author{
J. Paley, W. Badgett
}

Beam used: LArIAT Tertiary Beam, cosmics

Run dates: $7 / 25 / 2016$ - present

Prototype large-area picosecond photodetectors (pLAPPDs), developed at Argonne National Laboratory (ANL), present an opportunity to provide identification of charged particles in the 2-10 GeV/c range. Traditional methods using ionization or Cherenkov detectors require large amounts of material or pathlength for the particle to traverse in order to get a clean measurement, and are ill suited for particle beam identification in fixedtarget experiments. The $6 \times 6 \mathrm{~cm}^{2} \mathrm{pLAPPD}$ uses mm-thick borosilicate glass micro-channel plates with a resistive surface coating added via atomic layer deposition. Electrons emitted by the photocathode are accelerated through the MCPs, which have $\mu \mathrm{m}$-sized capillaries, and an avalanche of electrons is created as the electrons collide agains the walls of the capillaries of the MCPs. The resulting electron avalanche is collected on anode strip-lines and the signal may be read out on both ends of the strip-line. The resistive coating of the thin MCPs and the short pathlength of the electrons to the readout results in fast rise times, gains of greater than 106 and typical timing resolutions of 50 ps. Differential timing on the ends of the strip-lines can provide mm-level position measurements. The material of the bare pLAPPD presents less than 0.05 of an interaction length and approximately 0.12 of a radiation length for a charged particle of $1 \mathrm{GeV} / \mathrm{c}$. In our application, two pLAPPDs separated by 5-10 meters can be used in a time-of-flight (ToF) measurement for $3 \sigma$ separation of 2-7 GeV/c charged pions, kaons and protons with minimal impact on the particle's trajectory and momentum. The goal of this test-beam study is to measure the efficiency, timing resolution and position resolution the pLAPPDs using both FTBF beam, as shown in Figure 32 and cosmic rays using the FTBF cosmic ray telescope, as depicted in Figure 33.

\section{Setup}

A pLAPPD was installed in the LArIAT tertiary beamline as shown in the photo in Figure 32 , only a few days before the 2016 accelerator shutdown, and read out via the LArIAT DAQ. Unfortunately it discovered too late that we were looking at the incorrect electronics channel in the LArIAT data quality monitor, and that we were in fact getting no signals from the device. Upon investigation of the pLAPPD board, it was discovered that key 
components (a resistor and a capacitor) were missing from the board and there was no HV across the MCPs in the device. Around the time this was discovered, the beam turned off.

During the accelerator shutdown, another pLAPPD was inserted in the cosmic ray test stand (CRTS), in the location labeled "LAPPD 1" in Figure 33. This device was tested and the single-pe noise signals were recorded in a digital oscilloscope (see Figure 34). The signals from this device were then recorded in a CAEN VME waveform digitizer (V1751, borrowed from the LArIAT experiment) using the LArIAT DAQ, and trigger signals formed from coincident signals between the top and bottom scintillators and all four wire chambers in the CRTS. Data were collected with three different configurations of radiators: bare pLAPPD, pLAPPD+thin plastic scintillator and pLAPPD $+5 \mathrm{~mm}$ thick fused quartz block. When beam returned, the pLAPPD was again installed in the upstream end of the LArIAT tertiary beam-line. The V1751 was needed to be returned to the LArIAT experiment, and so we attempted to record digitized signals from the pLAPPDs using a new V1742 device. The data from this setup have not yet been analyzed, but a preliminary investigation indicates that there were some timing issues with these electronics that will significantly complicate the analysis.

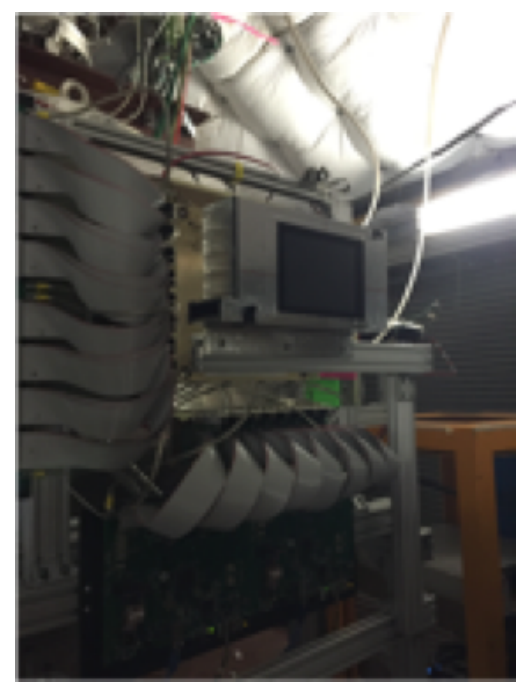

Figure 32: pLAPPD in he LArIAT Tertiary beamline.

\section{Results and Publications}

Signals produced by particles traversing the length of the CRTS and passing through the pLAPPD were successfully recorded in the V1751. Figure 35 shows the average pulse shape recorded for CRTS-triggered events. These data were used to determine relative efficiencies between the three configurations of radiators. We found that placing a thin piece of plastic 


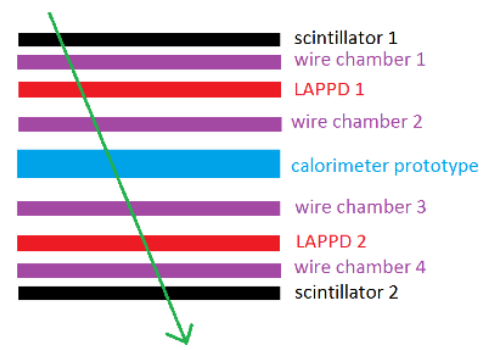

Figure 33: pLAPPDs in cosmic ray telescope at the FTBF.

scintillator in front of the pLAPPD glass window increased the efficiency by approximately $10 \%$. The efficiency increased another $20 \%$ when the scintillator was replaced with a piece of $5 \mathrm{~mm}$-thick fused quartz.

The impact of these results are two-fold: we have demonstrated that we can successfully record signals from the pLAPPD using the artdaq-based LArIAT DAQ, and the boardreader code used will be easy to port over to the artdaq-based protoDUNE-SP DAQ. We also demonstrated that we can significantly increase the signal efficiency by adding extra layers of fused quartz in front of the pLAPPD.

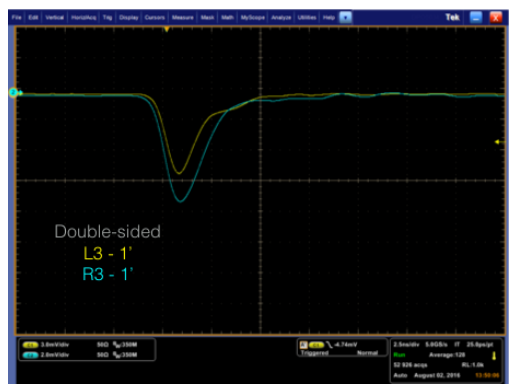

Figure 34: Digital oscilloscope screen capture of a single photoelectron noise signal read out on both ends of a stripline in a pLAPPD. 


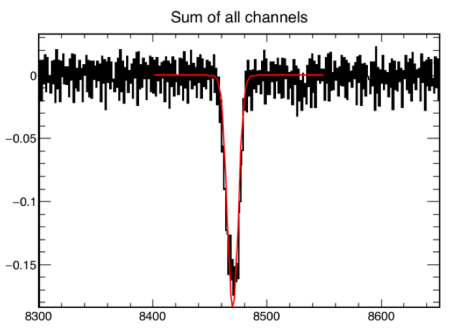

Figure 35: Average pLAPPD pulse shape recorded in a CAEN V1751 waveform digitizer for CRTS-triggers events. Signal pulses found at this time were used to calculate relative efficiencies between the three different configurations of radiators.

\title{
20 T1314: Fast Hadronic Calorimetry
}

\section{Motivation and Goals}

\author{
D. Denisov, S. Lukić, P. Ujić
}

Beam used: $p^{+} 120 \mathrm{GeV}, \pi^{+} 60 \mathrm{GeV}, \pi^{+} 30 \mathrm{GeV}, \pi^{+} 30 \mathrm{GeV}$ in muon mode Run dates: 4/21/2017 - 6/2/2017

Detector systems at future colliders will face the challenge of event rates and background levels substantially higher than in previous generations of experiments. Among the most important experimental criteria for the separation of pileup and background is the time of the detected energy deposits. To profit from the time information, integration times must be as short as possible in all detector subsystems. For the hadronic calorimetry, very short integration times cause a degradation of energy resolution because of the late component of hadronic showers. Depending on the absorber material, hadronic showers may continue developing up to $\sim 2 \mu s$.

FastHCal is an experiment to measure the fraction of deposited energy of hadronic showers within the first several nanoseconds of the shower development. This data will be used as benchmark for simulations to assess the deterioration of the energy resolution resulting from very short integration times.

\section{Setup}

The measurement setup was installed in the MTest section $6.2 \mathrm{C}$, on the remotely controlled motion table. The setup is schematically presented in Figure. 36. The calorimeter prototype consists of two iron absorbers with a cross section of $30 \times 30 \mathrm{~cm}^{2}$. The absorbers are composed of iron tiles so that the thickness of the front and the back absorber can be 
configured in various ways. Care was taken to avoid longitudinal cracks in the iron contruction along and near the beam axis. The density of the iron pieces was measured to be consistent with the density of iron within the $2 \%$ uncertainty of the weighting instrument used. The total thickness of the iron absorber is $60 \mathrm{~cm}$, corresponding to $3.6 \lambda_{\text {int }}$.

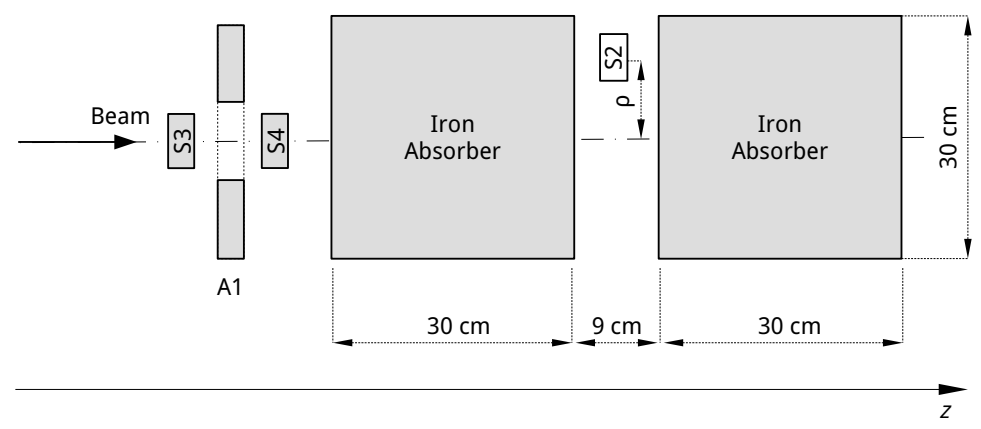

Figure 36: Sketch of the FastHCal setup (top view).

Scintillation counter S2 is positioned between the absorbers to measure local time development of energy deposit from hadronic showers. Counter S2 has a cross section of $2.5 \times 15 \mathrm{~cm}^{2}$ and a thickness of $1.2 \mathrm{~cm}$. It is mounted in the vertical position at distance $\rho$ from the beam axis. The installation and the dimensions of counter S2 are sketched in Fig. 37.

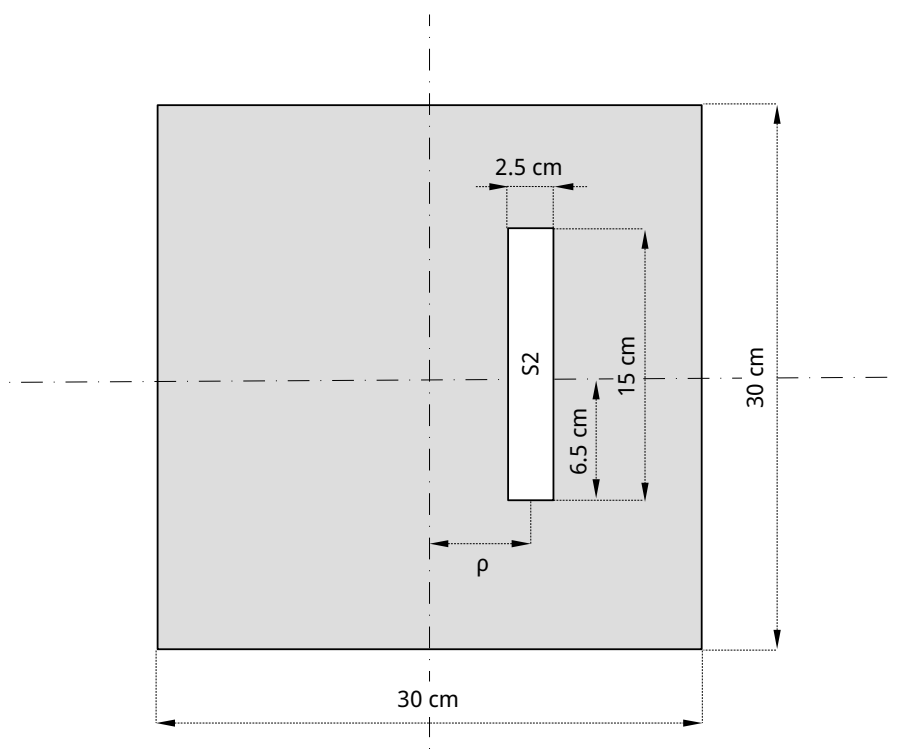

Figure 37: Sketch of the counter S2 and the absorber in the transverse plane. 
Upstream from the prototype, Counters S3, S4 and A1 are positioned on the beam axis to trigger the signal acquisition. The dimensions of counter S3 are $2.5 \times 18 \times 1.25 \mathrm{~cm}^{3}$, of counter S4 $2.0 \times 6 \times 1.1 \mathrm{~cm}^{3}$ and of counter A1 $25.5 \times 25.5 \times 1.0 \mathrm{~cm}^{3}$. The dimensions are given in the horizontal direction perpendicular to the beam, vertical direction and along the beam, respectively. Counter A1 has a circular hole of $4 \mathrm{~cm}$ diameter centered on the beam axis to veto upstream showers. The beam diameter at $1 / 10$ of the maximum is $1 \mathrm{~cm}$. The trigger logic is $\mathrm{S} 3 \times \mathrm{S} 4 \times \overline{\mathrm{A} 1}$. Trigger signals are formed using NIM discriminator and coincidence modules.

MIP response of counter S2 was recorded periodically using the $120 \mathrm{GeV}$ proton beam with the iron absorbers moved out of the beam.

Data acquisition was performed using a DRS4 digitizer evaluaton board (www.psi.ch/drs). Signals from counters S2, S3 and S4 were digitized. Digitized signals of S3 and S4 were used to form the time reference for the passage of the beam particle $t_{\text {ref }}=\left(t_{3}+t_{4}\right) / 2$.

\section{Results and Publications}

The measured pulse shapes induced by the hadronic showers in counter S2 are averaged over a large event sample for comparison with measured average pulse shapes induced by MIP. Very small difference between the pulse shape from hadronic showers and the pulse shape from MIP is found, indicating that shower energy deposition is very fast. Time evolution of the shower energy deposit is obtained by deconvolution of the MIP response from the average pulse shapes for different hadronic beam energies. Fig. ?? shows an example of the extraction of the time dependence of deposited energy.

Submission to NIM A is planned. 


\title{
21 T1315: LBNF Spectrometer, a preliminary duty cycle estimate with real beam
}

\section{Motivation and Goals}

\author{
P. L.G. Lebrun, L. Fields, D. Christian, R. Zaki
}

Beam Used: $120 \mathrm{GeV}$ Protons

Run Dates: May 3 to June 20, 2017

The LBNF Spectrometer project, aimed at determining the flux of neutrino progenitors, will require a low to medium intensity beam of $120 \mathrm{GeV}$ protons. Optimally, we want on average one proton per $53 \mathrm{MHz}$ r.f. bucket, and take data during the $\approx 50 \mu \mathrm{s}$ horn pulse. Unfortunately, the presence of "super-buckets" in the beam is likely to degrade the effective DAQ rate (or duty cycle) by possibly large factors. The MTest beam-line is well suited to quantitatively determine the time structure of the slow extracted beam at a typical Fermilab Fixed target beam-line and to develop the detector and DAQ to characterize this duty cycle.

\section{Setup}

Three plastic scintillator counters were installed in the MT6.1b area. A picture of the setup is shown on Figure 38. The front-end electronic was conventional (Lecroy modules). Waveforms from the PMT signals were recorded by a DRS4 module provided by the facility. Counting rates were bench-marked against the facility counter MT6SC1.

The DRS4 waveform allowed us to calibrate the one Minimum Ionization Particle energy loss. Based on this crude but effective calibration, discriminator threshold were set and coincidence signals were generated. Such coincidence triggers (efficiency better than $\approx 95 \%$ ) were recorded with a NimPlus/CAPTAN DAQ module, a cooperative effort between the Particle Physics Division and Scientific Computing Divions. For each spill, we determined the time of arrival for each particle in the beam with a precision of about $1 \mathrm{~ns}$, during the 4.1 second main injector spill.

We typically took beam half of one shift at a time. Often, we did this parasitically. We had dedicated beam on a week-ends (Memorial Day week-end), or off-hours, during the month of June and early July. A total of about 6 shifts were taken. We always used 120 $\mathrm{GeV}$ proton, with intensities ranging from 5,000 counts on MT6SC1, up to the maximum allowed of $\approx 1.510^{6}$. 


\section{Results and Publications}

Based on NimPlus/Captan data sets, one can compute an effective duty cycle for an arbitrary DAQ "time unit", requiring one and only proton per such time units. Such time unit range from a millisecond to a fraction of one microsecond, for the range of beam intensity used at MTest. (For the real LBNF spectrometer, we hope to build a $20 \mathrm{MHz}$ DAQ/trigger). This duty cycle factor is defined as the mean of the probabilities to get one and only trigger per such time unit. Should the instantaneous intensity be perfectly uniform during the entire spill, the optimum DAQ rate (the inverse of our time unit) would be such that the mean time unit occupation be close to 1, corresponding to a duty cycle factor of 0.36, based on the well known Poisson distribution. Variations of the intensity during spill, occurring one time scales ranging from tens of microseconds to seconds, do indeed reduce the duty cycle factor, as shown on Figure 39, where the duty cycle factor for hypothetical DAQ rates. Instead of a "perfect" duty cycle factor of 0.36 , we got only about 3 to $10 \%$. This was obtained running close to the maximum allowed intensity at M-Test.

We plan to write a DUNE DocDB note and work on the design of the LBNF spectrometer during F.Y. 18 - F.Y.20.

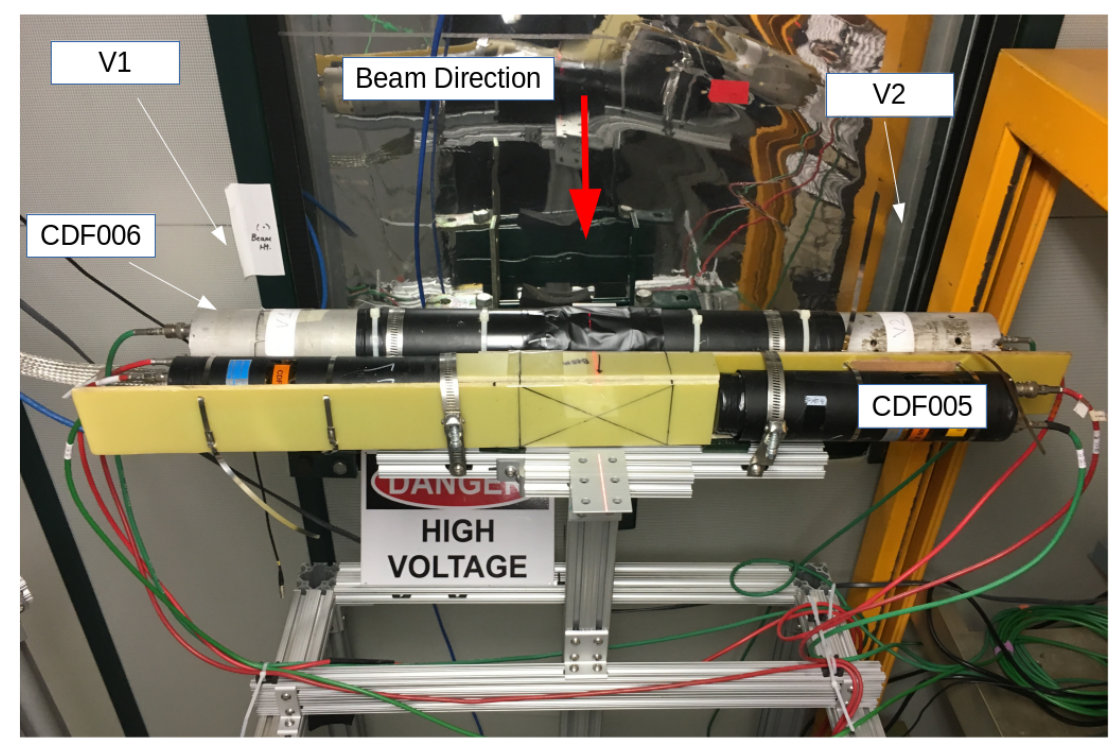

Figure 38: Picture of our setup at the area MT6-1b. The camera was place behind the counters, above the beamline and shooting at $\approx 20$ degree downwards (credit: Rowan Zaki) 


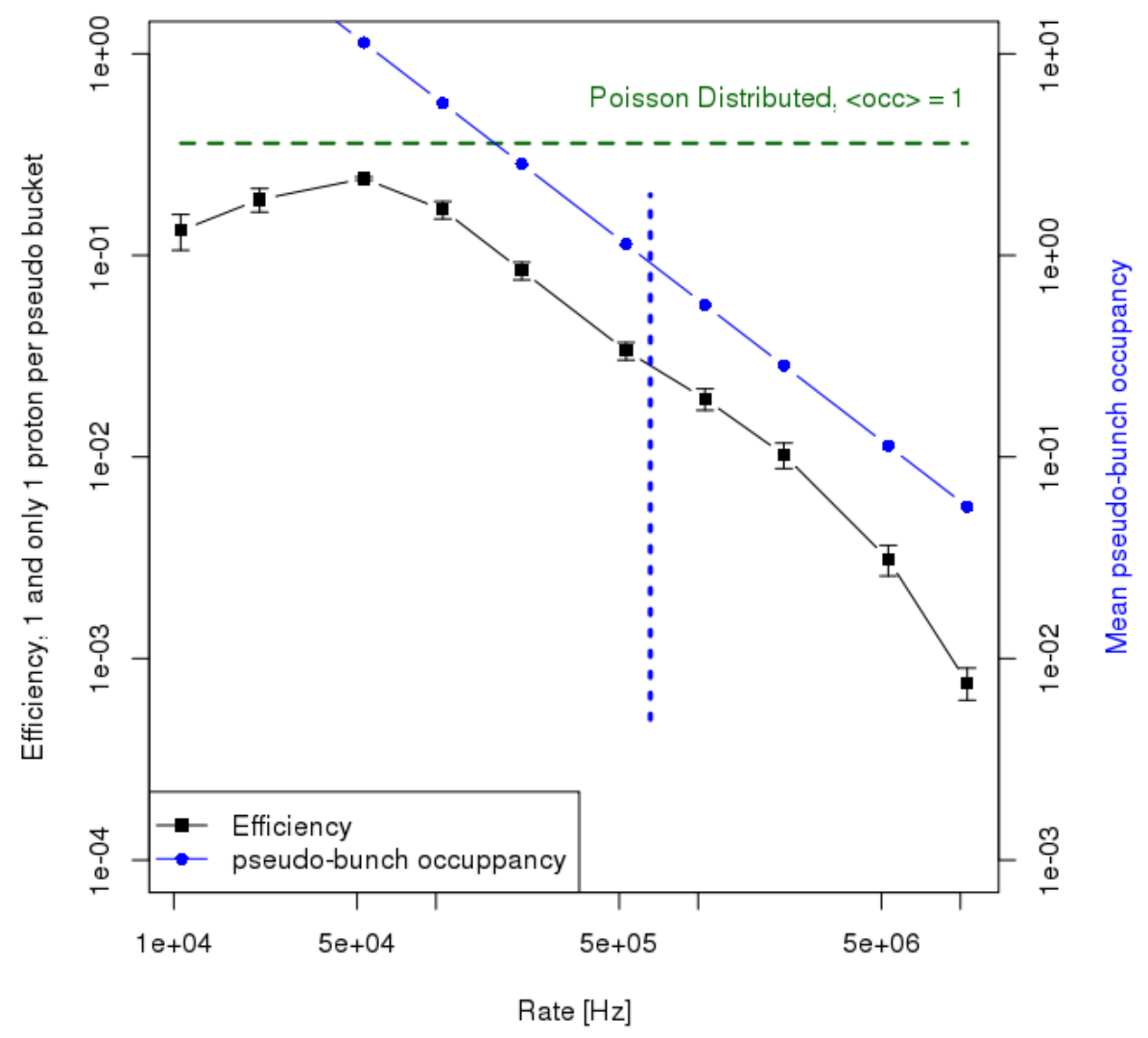

Figure 39: The efficiency, or duty cycle factor vs assumed DAQ rate, based on NimPlus/CAPTAN trigger timing data, obtained at a MT6SC1 count of $1.410^{6}$ per spill. Also shown is the mean time unit occupancy. In our case, should be spill be perfectly uniform, the optimum DAQ rate would be $\approx 610^{5} \mathrm{~Hz}$, instead, it is about one order of magntitude lower.

\section{A Publications}

T992: Tests of Radiation-hard Sensors for the HL-LHC

- Internal notes in preparation.

\section{T1041: CMS Forward Calorimetry R\&D}

- Internal CMS note 


\section{T1043: Mu2e CRV Test Beam}

- Publication of the full test beam results is pending.

\section{T1044: sPHENIX Calorimeter Test}

- "Design and Beam Test Results for the sPHENIX Electromagnetic and Hadronic Calorimeter Prototypes", sPHENIX collaboration. Submitted to IEEE Transactions on Nuclear Science. arXiv:1704.01461

- FY17 test beam paper in preparation for publication

\section{T1065: Fast Timing Detectors}

- Preparing article for NIM for publication

\section{T1068: Beam Test of the SVX4 Telescope}

- Wating on information from spokesperson.

\section{T1209 CMS OuterTracker}

- Results are in the HL-LHC TDR, under preparation

\section{T1224: ATLAS Pixel}

- RTI Pixel Module Evaluation, ATLAS internal note, in preparation.

- Testbeam measurements of pixel sensor technologies for ITK upgrade, Dylan Frizzell, presentation at the US ATLAS Workshop, Argonne National Laboratory, July 2017.

- Track Reconstruction Efficiencies with the H35DEMO HV-CMOS Pixel Detector, Matt Zhang, presentation at the American Physical Society Division of Particles and Fields, Fermi National Accelerator Laboratory, August 2017.

- Track Reconstruction Efficiencies with the H35DEMO HV-CMOS Pixel Detector, Dylan Frizzell, poster at the American Physical Society Division of Particles and Fields, Fermi National Accelerator Laboratory, August 2017.

\section{T1267: IceCube DOM Test}

- Used in internal simulations

\section{T1272: Prototype EM Calorimeter}

- "A LYSO crystal mini-calorimeter readout by silicon photo-multipliers as compact detector for space applications", A. Kryemadhi, L. Barner, A. Grove, J. Mohler, A. Roth, Poster presented at NDIP17, France. 


\section{T1273: LHCb VELO Beam Test}

- Report in preparation

\section{T1314: Fast Hadronic Calorimetry}

- Planned submission to NIM A

T1315: LBNF Spectrometer, a preliminary duty cycle estimate with real beam

- Internal DUNE note 